ARTICLE

\title{
Spectroscopic evidence for a large spot on the dimming Betelgeuse
}

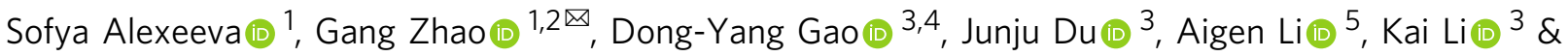 \\ Shaoming $\mathrm{Hu}$ (iD) $^{3}$
}

During October 2019 and March 2020, the luminous red supergiant Betelgeuse demonstrated an unusually deep minimum of its brightness. It became fainter by more than one magnitude and this is the most significant dimming observed in the recent decades. While the reason for the dimming is debated, pre-phase of supernova explosion, obscuring dust, or changes in the photosphere of the star were suggested scenarios. Here, we present spectroscopic studies of Betelgeuse using high-resolution and high signal-to-noise ratio nearinfrared spectra obtained at Weihai Observatory on four epochs in 2020 covering the phases of during and after dimming. We show that the dimming episode is caused by the dropping of its effective temperature by at least $170 \mathrm{~K}$ on 2020 January 31, that can be attributed to the emergence of a large dark spot on the surface of the star.

\footnotetext{
${ }^{1}$ CAS Key Laboratory of Optical Astronomy, National Astronomical Observatories, Chinese Academy of Sciences, Beijing, China. ${ }^{2}$ School of Astronomy and Space Science, University of Chinese Academy of Sciences, Beijing, China. ${ }^{3}$ Shandong Key Laboratory of Optical Astronomy and Solar-Terrestrial Environment, Institute of Space Sciences, School of Space Science and Physics, Shandong University, Weihai, China. ${ }^{4}$ School of Astronomy and Space Science, Key Laboratory of Ministry of Education, Nanjing University, Nanjing, China. ${ }^{5}$ Department of Physics and Astronomy, University of Missouri, Columbia, MO, USA. $\varpi_{\text {email: gzhao@nao.cas.cn }}$
} 
B etelgeuse ( $\alpha$ Orionis) is one of the closest luminous red supergiants (RSGs) to the Earth and due to its brightness it can be seen by naked-eye in the night sky. Betelgeuse appears to be on the late evolutionary stage of massive stars and sooner or later it will explode as a Type II-P Supernova and turn into a relativistic compact object. It is promising to offer us an amazing celestial show. Such events occur rarely in the human era therefore at present Betelgeuse attracts not only professional astronomers, but also has been widely covered in mass media. For astronomers, it is an important astronomical laboratory for the investigation of stellar evolution, stellar atmosphere, presupernova explosion, and dust condensation.

Betelgeuse is classified as a semi-regular variable star with spectral type M2 Lab. During the period from October 2019 to mid-March 2020, Betelgeuse demonstrated a deep minimum of its brightness. On 2019 October 25, its visual $V$ magnitude was +0.679 mag (Universal Time (UT) 11.06). After that, it was losing its luminosity with an average rate of 0.01 magnitudes per day, reaching the minimum on 2020 February $9(V=+1.638$, UT 23.33) and it is the deepest one within the past 8 years. However, already in March 2020 , Betelgeuse was rapidly recovering luminosity at an average rate of 0.02 magnitudes per day and on 2020 April 19, it became even brighter than the day before dimming started $(V=+0.487$, UT 02.57). One of the interesting features of this great dimming is that the rate of brightening of Betelgeuse $(\sim 0.02 \mathrm{mag} /$ day $)$ is two times higher than the rate of its dimming $(\sim 0.01 \mathrm{mag} / \mathrm{day})$. This rebrightening event has also led to intense discussions in the science community. To explain this minimum of its brightness, several explanations have been proposed: pre-phase of the supernova explosion, obscuring dust, or changes in the photosphere of the star.

The European Southern Observatory's (ESO) Very Large Telescope (VLT) reported about the unusual dimming of Betelgeuse. The images of the star's surface not only show it losing luminosity but also its shape seemingly changing ${ }^{1}$. They presented the comparison image of Betelgeuse before and after its dimming. The observations, taken with the Spectro-Polarimetric High-contrast Exoplanet REsearch (SPHERE) instrument on ESO-VLT in January and December 2019, show how much the star has faded and how its apparent shape has changed.

The spectrophotometric study of Betelgeuse reported in ref. ${ }^{2}$ suggested that the effective temperature $\left(T_{\text {eff }}\right)$ on 2020 February 15 was $T_{\text {eff }}=3600 \pm 25 \mathrm{~K}$ and the derived small drop $\Delta T_{\text {eff }}=50 \mathrm{~K}$ was insufficient to explain the observed dimming. Since RSGs like Betelgeuse are surrounded by a gaseous and dusty circumstellar envelope created by their mass loss, which spreads heavy elements into the interstellar medium, it was proposed that the recent dimming was caused by screening from circumstellar dust. The possible chemical composition of the dust and the normalized masses of the dust grains that could condense around the cooling envelope of Betelgeuse and screen the light was predicted in ref. ${ }^{3}$.

During September, October, and November 2019, three months before the dimming event, the ultraviolet spectroscopic observations from the Hubble Space Telescope (HST)/Space Telescope Imaging Spectrograph (STIS) revealed an increase in the ultraviolet spectrum and $\mathrm{Mg}$ II line emission from the chromosphere of the Betelgeuse ${ }^{4}$. The detected variations in the $\mathrm{Mg}$ II $\mathrm{k}$-line profiles gave evidence of the movement of material outwards. This movement, likely due to convective photospheric elements, was further aided with outward motions during the $\sim 400$-day pulsation cycle. A relationship between the large convective cells and material movement was inferred via ultraviolet observations and suggested that the ejected material cooled as a dust cloud and likely caused the exceptional optical dimming of Betelgeuse in February $2020^{4}$.

However, only small changes in the gas velocities were detected from the high spectral-resolution observations of [Fe II ] $25.99 \mu \mathrm{m}$ and [S I ] $25.25 \mu \mathrm{m}$ emission lines from Betelgeuse obtained with the Stratospheric Observatory for Infrared Astronomy (SOFIA) Echelon Cross Echelle Spectrograph (EXES) during the dimming event on February 2020. It was argued that the dimming could not be due to dust gave the lack of variation in the outflow velocities and line ratios ${ }^{5}$.

The TiO (titanium oxide) and near-infrared (near-IR) photometric data obtained at the Wasatonic Observatory ${ }^{6}$ found a significantly cooler mean effective temperature of Betelgeuse during the dimming event than that inferred from the spectrophotometric observations reported in ref. ${ }^{2}$.

Submillimetre (sub-mm) observations from the James Clerk Maxwell Telescope (JCMT) and Atacama Pathfinder Experiment (APE) over a period of 13 years which includes the dimming period, indicates a decline in sub-mm flux by $20 \%$ compared to values before the dimming event ${ }^{7}$. The modeling presented in ref. ${ }^{7}$ indicates that the condensed dust cannot be the reason for this sub-mm flux drop, and only changes in the photosphere of a star (change in radius or temperature) might lead to the sub-mm flux reduction.

An alternative scenario was presented by George et al. ${ }^{8}$, who suggested that the dimming of Betelgeuse might be caused due to critical transition in the pulsation dynamics.

Here, we present the spectroscopic study of Betelgeuse with the high-resolution and high signal-to-noise ratio $(S / N)$ spectra obtained at Weihai Observatory of Shandong University on 2020 January 31, March 19, April 4, and April 6. We determine the effective temperature $\left(T_{\text {eff }}\right)$, surface gravity $(\log g)$, and microturbulence $\left(V_{\text {mic }}\right)$ of Betelgeuse by employing a Markov Chain Monte Carlo (MCMC) forward-modeling method. We rule out pulsations as a possible mechanism of temperature decreasing and confirm that this dimming episode is caused by the dropping of its effective temperature by at least $170 \mathrm{~K}$ on 2020 January 31, which can be attributed to an emergence of a large dark spot on the surface of the star. We deduce the chemical compositions of $\mathrm{C}, \mathrm{N}, \mathrm{O}, \mathrm{Na}, \mathrm{Mg}, \mathrm{Ca}, \mathrm{Ti}, \mathrm{Cr}, \mathrm{Fe}$, and $\mathrm{Sr}$ at the pre-dimming, dimming, and post-dimming episodes and show that the chemical abundances are stable in time if the variable temperature is considered. Our results are consistent with ESOVLT $^{1}$, ultraviolet HST-STIS ${ }^{4}$, infrared SOFIA-EXES ${ }^{5}$, and JCMT submillimeter ${ }^{7}$ observations that the dimming of Betelgeuse is unlikely caused by dust obscuration.

\section{Results}

Effective temperature. The physical parameters of Betelgeuse in the pre-dimming, dimming and post-dimming epochs are presented in Table 1 and Fig. 1a-d. We have found that on 14-02-2012, when the visual magnitude $V=0.426$, the effective temperature of Betelgeuse was $T_{\text {eff }}=3632 \pm 7 \mathrm{~K}$. During the dimming on 31-01-2020, when $V$ $=1.609$, the effective temperature was found to be lower by $156 \mathrm{~K}$ compared to pre-dimming episode. After 31-01-2020, the $T_{\text {eff }}$ was increasing and became $T_{\text {eff }}=3534 \pm 3 \mathrm{~K}$ on 19-03-2020, then $T_{\text {eff }}=$ $3611 \pm 7 \mathrm{~K}$ on $04-04-2020$, and finally reached $T_{\text {eff }}=3646 \pm 9 \mathrm{~K}$ on 06-04-2020, which is close to its pre-dimming temperature. The effective temperature was found to be well correlated with the visual magnitude at all epochs of observations (Fig. 1a, c).

The changes in $T_{\text {eff }}$ are sufficient to explain the recent variations in $V$ magnitude. To demonstrate this, we adopted the formula for the $V$-band bolometric correction, which was computed by employing MARCS stellar atmosphere models ${ }^{9-13}$ :

$$
\begin{array}{r}
\mathrm{BC}_{\mathrm{V}}=-298.954+217.532 \frac{T_{\text {eff }}}{1000(\mathrm{~K})} \\
-53.14\left(\frac{T_{\mathrm{eff}}}{1000(\mathrm{~K})}\right)^{2}+4.34602\left(\frac{T_{\mathrm{eff}}}{1000(\mathrm{~K})}\right)^{3} .
\end{array}
$$

According to (1), a rise of $170 \mathrm{~K}$ in $T_{\text {eff }}$ from $3476 \mathrm{~K}$ of $31-01$ 2020 to $3646 \mathrm{~K}$ of $06-04-2020$ would correspond to a decrease in 
Table 1 Physical parameters of Betelgeuse obtained in this study.

\begin{tabular}{|c|c|c|c|c|c|c|c|c|}
\hline Date of observations & JD-2450000 (d) & $T_{\text {eff }}(K)$ & $\sigma\left(T_{\text {eff }}\right)$ & $\log g$ & $\sigma(\log g)$ & {$[\mathrm{Fe} / \mathrm{H}]$} & $\sigma([\mathrm{Fe} / \mathrm{H}])$ & $V_{\text {mic }}\left(\mathrm{km} \mathrm{s}^{-1}\right)$ \\
\hline $06-04-2020$ & 8945.6 & 3646 & 9 & 0.24 & 0.04 & 0.11 & 0.07 & 3 \\
\hline $04-04-2020$ & 8943.6 & 3611 & 7 & 0.17 & 0.04 & 0.12 & 0.15 & 3 \\
\hline $19-03-2020$ & 8927.6 & 3534 & 3 & 0.25 & 0.04 & 0.06 & 0.09 & 3 \\
\hline $14-02-2012$ & 6702.6 & 3632 & 7 & 0.11 & 0.03 & 0.07 & 0.16 & 3 \\
\hline
\end{tabular}

Julian date (JD-2450000), effective temperature $\left(T_{\text {eff }}\right)$, surface gravity $(\log g)$, metallicity $([\mathrm{Fe} / \mathrm{H}])$, and microturbulent velocity $\left(V_{\text {mic }}\right)$ ) are presented. Date of observations is given in dd-mm-yyyy format. Uncertainty for $T_{\text {eff }}$ and $\log g\left(\sigma\left(T_{\text {eff }}\right)\right.$ and $\left.\sigma(\log g)\right)$ is defined as the difference between the 84th and 50th percentile as the upper limit, and the difference between the 50th and 16th percentile as

the lower limit. Uncertainty for $[\mathrm{Fe} / \mathrm{H}](\sigma([\mathrm{Fe} / \mathrm{H}]))$ is calculated as standard deviation. Source data are provided as a Source Data file.

a

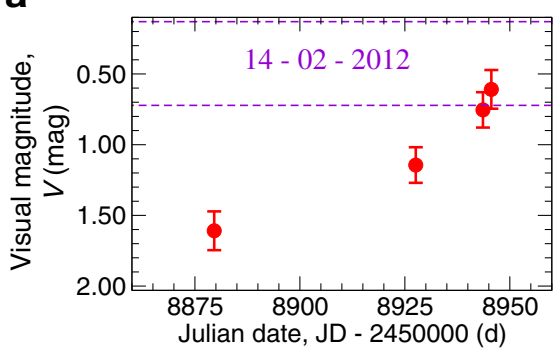

C

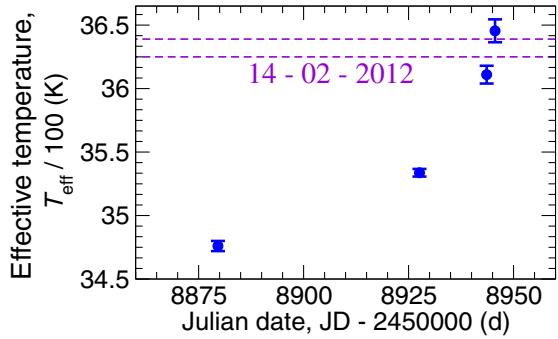

b

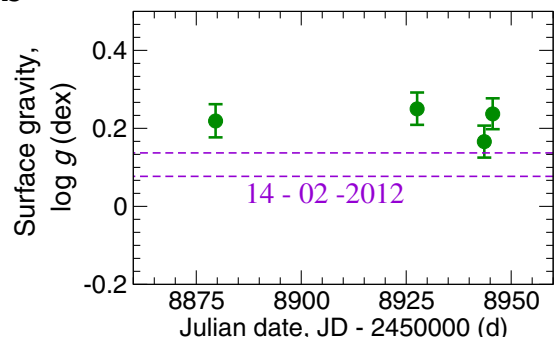

d

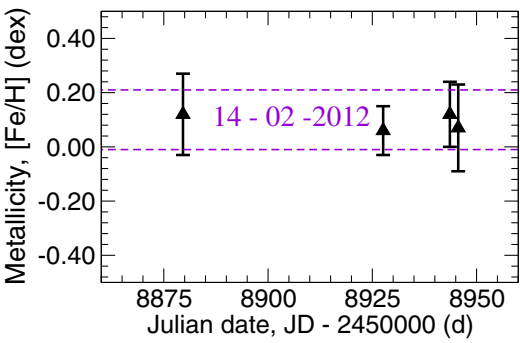

Fig. 1 The mean visual magnitude $(V)$, surface gravity $(\log g)$, effective temperature $\left(T_{\text {eff }}\right)$, and metallicity ([Fe/H]) of Betelgeuse obtained on 2020 January 31, March 19, April 4, and April 6. a Each V magnitude was calculated by averaging over all available magnitudes in the AAVSO database in a particular date of observations. b The uncertainty of surface gravity $(\log g)$ is taken as the difference between the 84th and 50th percentile as the upper limit, and the difference between the 50th and 16th percentile as the lower limit. c The uncertainty of the effective temperature ( $T_{\text {eff }}$ ) is taken as the difference between the 84th and 50th percentile as the upper limit, and the difference between the 50th and 16th percentile as the lower limit. $\mathbf{d}$ Metallicity $([\mathrm{Fe} / \mathrm{H}])$ was calculated by averaging over all iron abundances from individual lines in a particular date of observations. The two horizontal dashed lines show the ranges of parameters $\left(V, T_{\text {eff }} \log g,[\mathrm{Fe} / \mathrm{H}]\right)$ for pre-dimming, which we have obtained with ESPaDOnS's spectrum observed on 2012 February, 14. Error bars represent standard deviation. Source data are provided as a Source Data file.

the $V$ magnitude by $\Delta V \approx 0.753 \mathrm{mag}$. This is fairly sufficient to account for the observed brightening of $\Delta V=0.918 \pm 0.171 \mathrm{mag}$, within the uncertainty. Similarly, a rise in $T_{\text {eff }}$ from $3476 \mathrm{~K}$ of 31 01-2020 to $3534 \mathrm{~K}$ of 03-19-2020 would lead to a decrease of $\Delta V \approx$ $0.301 \mathrm{mag}$, which, within the uncertainty, is also fairly enough to explain the observed brightening of $\Delta V=0.456 \pm 0.186 \mathrm{mag}$, and a rise in $T_{\text {eff }}$ from $3534 \mathrm{~K}$ of $19-03-2020$ to $3646 \mathrm{~K}$ of $06-04-2020$ would cause a decrease of $\Delta V \approx 0.452 \mathrm{mag}$. This closely agrees with the observed brightening of $\Delta V=0.453 \pm 0.162 \mathrm{mag}$.

Evidence for a large cool spot. What could lead to a temperature drop by $170 \mathrm{~K}$ ? It should be noted that the lowest temperature obtained on $31-01-2020, T_{\text {eff }}=3476 \mathrm{~K}$, would be one of the coolest effective temperatures measured for a Galactic RSG. Although unusual, an effective temperature as low as $3365 \pm 134$ $\mathrm{K}$ was found for the red supergiant UY Sct ${ }^{14}$. Among 74 Galactic RSGs, only one star cooler than $3500 \mathrm{~K}$ was found ${ }^{15}$. According to the stellar evolution theory, the Hayashi limit (the lower limit of $T_{\text {eff }}$ for stars in hydrostatic equilibrium) for a star like
Betelgeuse is $\sim 3500 \mathrm{~K}$ (see, e.g., Fig. 6.5 in ref. ${ }^{16}$ ). Nevertheless, the significance of the Hayashi limit for RSGs is still open to debate. It has been suggested that the stars moved to the forbidden region of the Hertzsprung-Russell (H-R) diagram are not able to maintain their hydrostatic equilibrium and this may actually drive episodes of enhanced mass loss. However, there are also some individual stars, which still have a $T_{\text {eff }}$ that places them to the right of the Hayashi limit, see e.g., ref. ${ }^{16}$. It is still unclear whether these stars are in fact in hydrostatic equilibrium (and their observational effective temperatures were misinterpreted), or truly challenge evolutionary theory with unexpected cold temperatures, or these stars have cool spots on the surface that decrease their average temperature.

We believe that it is unlikely that the entire Betelgeuse became cooler by $170 \mathrm{~K}$. It could be caused by a dark star spot on the surface of Betelgeuse. The presence of spots on the surface of Betelgeuse and other RSGs is a well-known phenomenon, see e.g., refs. ${ }^{17-21}$. These spots are likely a consequence of convective flows or cool convective cells, which are widely believed to be present in such stars ${ }^{22-25}$. The size of spots can probably be large. 
a

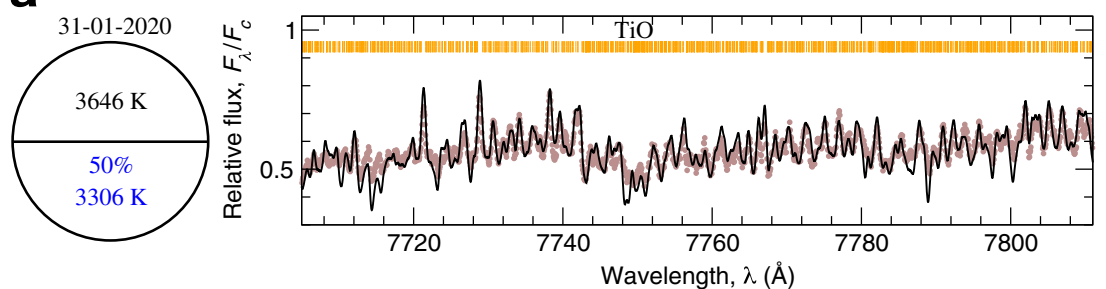

b
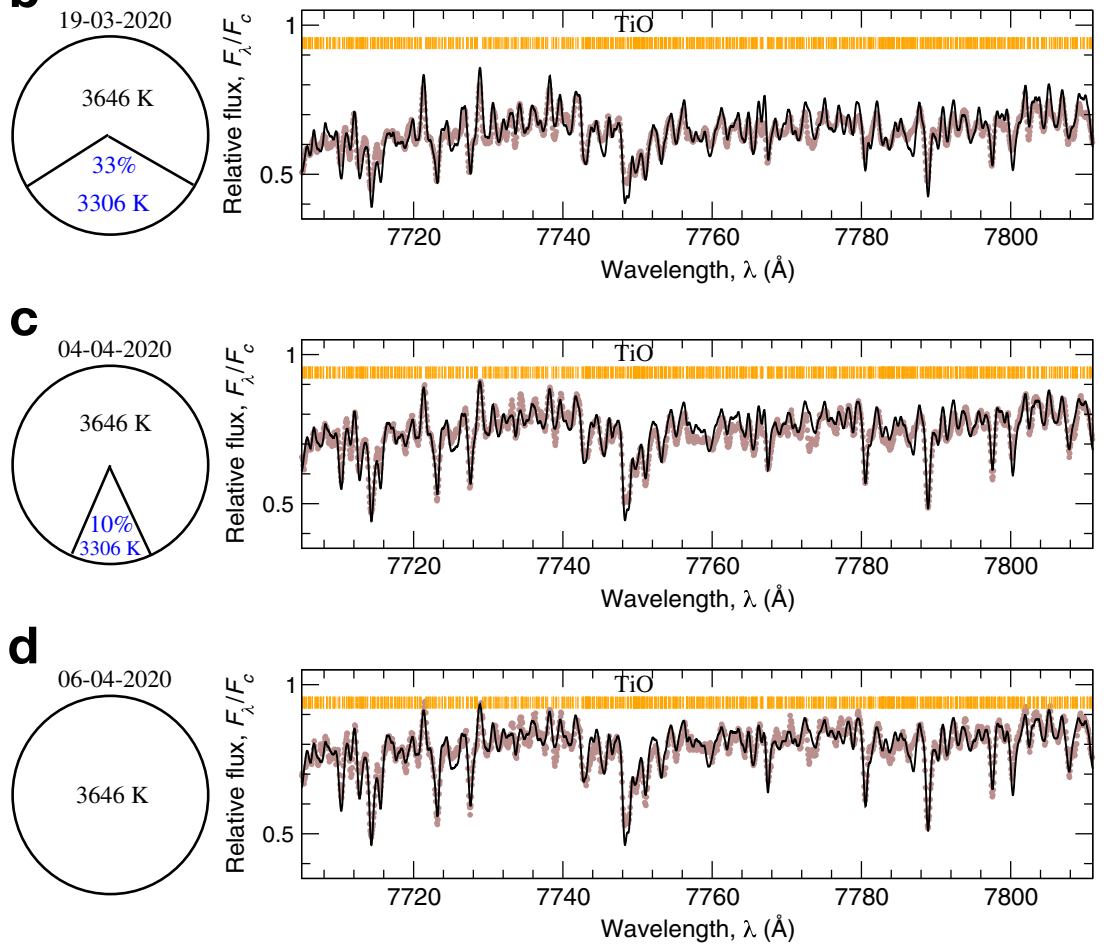

Fig. 2 The schemes of the stellar surface with a dark spot and the corresponding best fits of the narrow spectral region 7705-7811 Å for four dates of observations. The temperature increasing is interpreted as the changing of the surface area of a dark spot. Relative flux is calculated as a ratio of the flux at a particular wavelength $\left(F_{\lambda}\right)$ to the flux in continuum $\left(F_{c}\right)$. The observed spectra are shown as brown dots. The theoretical spectra (black solid curves) were calculated with the obtained parameters from Table 1 and convolved with the instrumental profile. Everywhere $V_{\text {mic }}=3 \mathrm{~km} \mathrm{~s}^{-1}, V \mathrm{sini}=5 \mathrm{~km} \mathrm{~s}-1, V_{\text {mac }}=$ $16 \mathrm{~km} \mathrm{~s}^{-1}$. Titanium, carbon, nitrogen, and oxygen abundances are taken to be 4.95, 8.43, 8.60, and 8.80 dex, respectively. The molecular TiO (titanium oxide) lines are marked by vertical lines on each panel. a On 31-01-2020, the temperature of the spot is $3306 \mathrm{~K}$ if it occupies $50 \%$ of the surface area. $\mathbf{b}$ On 19-03-2020, the temperature of a spot is $3306 \mathrm{~K}$ if it occupies $33 \%$ of the surface area. c On 04-04-2020, the temperature of a spot is $3306 \mathrm{~K}$ if it occupies $10 \%$ of the surface area. d On 06-04-2020, the temperature of the whole star is $3646 \mathrm{~K}$. Source data are provided as a Source Data file.

For example, $H$-band interferometric observations of Betelgeuse, showed two spots at the surface of the star and one of the spots is as large as one-half of the stellar radius ${ }^{26}$.

We present an interpretation of temperature deviation as a changing of the surface area of dark spots. The ESO-VLT observations $^{1}$ presented the images of the Betegeuse's surface before and after its dimming in January and December 2019 [https://www.eso.org/public/images/eso2003c/]. It is clearly seen, that about a half of the star has faded significantly. The dark region seen in the optical VLT observations could be explained by a large spot, whose temperature is lower than the typical temperature of Betelgeuse before the dimming. If we assume that in the minimum of brightness, on $31-01-2020,50 \%$ of the stellar surface was covered with dark spots, the temperature of spots would be $3306 \mathrm{~K}$. The dark-spot covered surface would change from $50 \%$ on $31-01-2020$ to $10 \%$ on $04-04-2020$. The scheme of stellar surface and the best fits of narrow spectral region 7705-7811 $\AA$ for four epochs are presented in Fig. 2a-d.

Taking into account its slow rotation velocity of $V \sin i=5.47$ $\mathrm{km} \mathrm{s}^{-1}$, the inclination of the polar axis on the line of sight $i=$ $60^{\circ}$ and a rotation period, $P / \sin i$, of 36 years $^{27}$, the star would rotate only by $\sim 2^{\circ}$ around its axis from 31-01-2020 to 06-04-2020. Such a small angle would not lead to a significant change of surface area due to the rotation of the star. We did not find any significant changes in surface gravity at all epochs of observations from 31-01-2020 to 06-04-2020, log $g$ remains constant within the error bars with a mean value $\log g=0.22 \pm 0.04$ (Fig. 1b). Although on 14-02-2012, the $\log g$ is slightly lower (by $0.11 \mathrm{dex}$ ), but still consistent within $3 \sigma$. If we assume a change in acceleration due to gravity as a result of a change in radius, $R$, (according to $g=\mathrm{GM} / R^{2}$ ), and take a $3 \sigma$ limit on the change of $\log g=3 * 0.04=0.12$, we can conclude that the $R$ could change at most $12 \%$ during the period of brightness recovering from 31-012020 to $06-04-2020$.

Even a small change in radius $(\sim 10 \%)$ at constant $T_{\text {eff }}$ would lead to a change in luminosity, $L$. If we know definitely that $T_{\text {eff }}$ decreased by $\sim 170 \mathrm{~K}$ and this decreasing is enough to explain changing in luminosity, it means that the radius of the star was definitely not changing much, because $L \sim T_{\text {eff }}^{4} R^{2}$. Otherwise, if both $T_{\text {eff }}$ and $R$ are decreasing simultaneously, it would lead to a 
deeper dimming. Another case, if $T_{\text {eff }}$ is falling down and $R$ is increasing simultaneously, then $L$ would not change at all and we would not observe any dimming (or we would observe brightening instead of dimming). Following these arguments, we can rule out pulsations as a possible reason for the dimming of Betelgeuse.

The microturbulent velocity was found constant during all the epochs and was estimated at $3 \mathrm{~km} \mathrm{~s}^{-1}$. We did not find any significant differences in the broadening of the lines, that can indicate a constant in the rotational velocity $(V \sin i)$, which was adopted to be $5 \mathrm{~km} \mathrm{~s}^{-1}$, according to ref. ${ }^{28}$. We have found overabundance in Fe relative to solar value at all epochs with average $[\mathrm{Fe} / \mathrm{H}]=0.09 \pm 0.03 \mathrm{dex}$ (Fig. 1d).

Chemical composition. We now elaborate the chemical composition of Betelgeuse with three different scenarios. The first scenario $\left(T_{\text {eff }}=\right.$ var, Figs. 3 and 4$)$ is that the effective temperature was changing according to our results presented in Table 1. According to this scenario, the chemical abundances of all considered elements, $\mathrm{C}, \mathrm{N}, \mathrm{O}, \mathrm{Na}, \mathrm{Mg}, \mathrm{Ca}, \mathrm{Ti}, \mathrm{Cr}, \mathrm{Fe}, \mathrm{Sr}$ remain constant within the error bars. This is reasonable since we do not expect the abundance of these elements change within such a short time span of $\sim 2$ months. In Fig. 3a, carbon abundances were obtained under the condition that $\mathrm{N}$ and $\mathrm{O}$ keep their constant values, $\mathrm{A}(\mathrm{N})=8.6$ and $\mathrm{A}(\mathrm{O})=8.8$. In Fig. 3b, nitrogen abundances were obtained at the condition that $\mathrm{C}$ and $\mathrm{O}$ keep their constant values, $\mathrm{A}(\mathrm{C})=8.43$ and $\mathrm{A}(\mathrm{O})=8.8$. In Fig. $3 \mathrm{c}$, oxygen abundances were obtained at the condition that $\mathrm{C}$ and $\mathrm{N}$ remain constant values, $\mathrm{A}(\mathrm{C})=8.43$ and $\mathrm{A}(\mathrm{N})=8.6$. In Fig. 4, we present the chemical abundances of the remaining elements. Everywhere, the solar abundances are shown as a horizontal dashed line.

The second scenario (Figs. 3 and 4, open squares) is the effective temperature was constant $\left(T_{\text {eff }}=3646 \mathrm{~K}\right)$ and $\log g=$ 0.2 was adopted. At the constant value of $T_{\text {eff }}$ at all five epochs, we are not able to reach the constant abundances of $\mathrm{C}, \mathrm{N}, \mathrm{O}, \mathrm{Mg}$ at these epochs. For example, in Fig. 3a, on 31-01-2020 (Julian date, JD-2450000 $=8879.6 \mathrm{~d}$ ), we would have $\mathrm{A}(\mathrm{C})=8.13$ (under condition $T_{\text {eff }}=3646 \mathrm{~K}, \mathrm{~A}(\mathrm{~N})=8.6$, and $\mathrm{A}(\mathrm{O})=8.8$ ), that is by 0.28 dex lower than at the epoch of 06-04-2020 (JD-2450000 = 8945.6 d). Another example, in Fig. 3b, on 31-01-2020 we would have $\mathrm{A}(\mathrm{N})=7.99$ (under condition $T_{\text {eff }}=3646 \mathrm{~K}, \mathrm{~A}(\mathrm{C})=8.43$, and $\mathrm{A}(\mathrm{O})=8.8$ ), that is by 0.61 dex lower than that at the epoch of 06-04-2020. In Fig. 3c, we would have $A(O)=9.12$ (under condition $T_{\text {eff }}=3646 \mathrm{~K}, \mathrm{~A}(\mathrm{C})=8.43$, and $\mathrm{A}(\mathrm{N})=8.6$ ), that is by 0.32 dex higher than at the epoch of 06-04-2020. Apparently, this is unlikely since it is difficult to imagine how the abundances of $\mathrm{C}$, $\mathrm{N}$, and $\mathrm{O}$ could considerably vary within the period of only $\sim 2$ months.

At the third scenario, we adopted the lowest temperature as a constant value during all epochs $\left(T_{\text {eff }}=3476 \mathrm{~K}\right.$, Figs. 3 and 4, open diamonds) and $\log g=0.2$. Analogically to the second scenario, we are not able to reach the constant abundances of $\mathrm{C}$, $\mathrm{N}, \mathrm{O}, \mathrm{Mg}$ at these epochs at the constant temperature. Here, we did not intend to analyze the absolute values of chemical abundances. We demonstrate that only if we assume the variable $T_{\text {eff }}$, we can reach consistency in the chemical composition of C, $\mathrm{N}, \mathrm{O}$, and $\mathrm{Mg}$ during all epochs of observations. As for other chemical elements, $\mathrm{Na}, \mathrm{Ca}, \mathrm{Ti}, \mathrm{Cr}, \mathrm{Fe}, \mathrm{Sr}$, it is impossible to conclude which scenario is more favorable, since the error bars are high.

Figure 5a-d presents the molecular CN (cyanide) line at 10,179 $\AA$, which was used along with others $\mathrm{CN}$ lines (Supplementary Table 1) for the estimation of $\mathrm{C}, \mathrm{N}, \mathrm{O}$ conditional abundances. The observed molecular CN line at 10,179 ̊ is stronger on 14-02-2012 compared
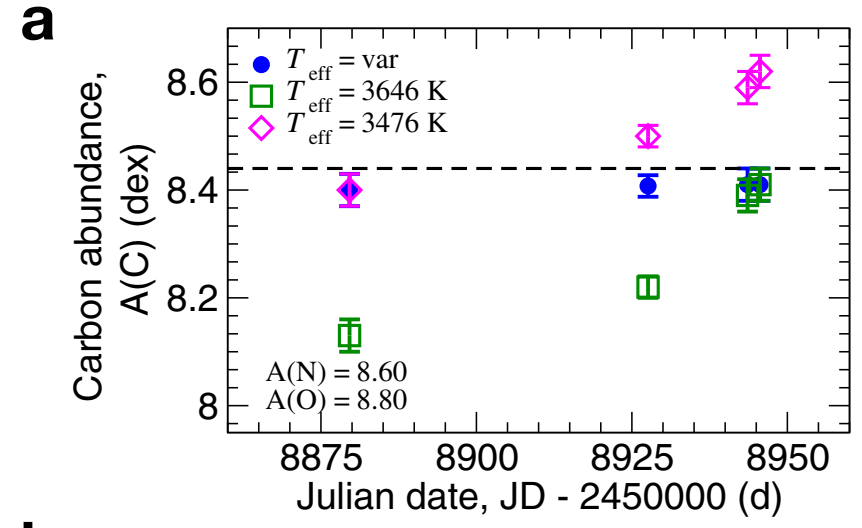

b

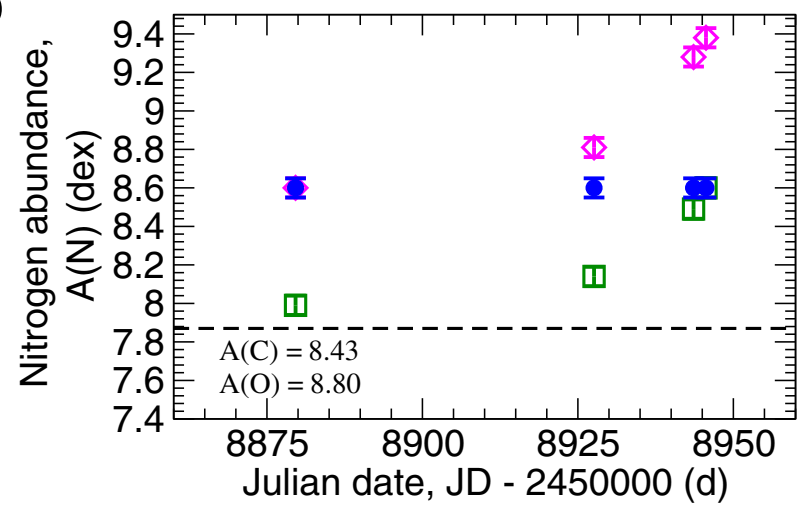

C

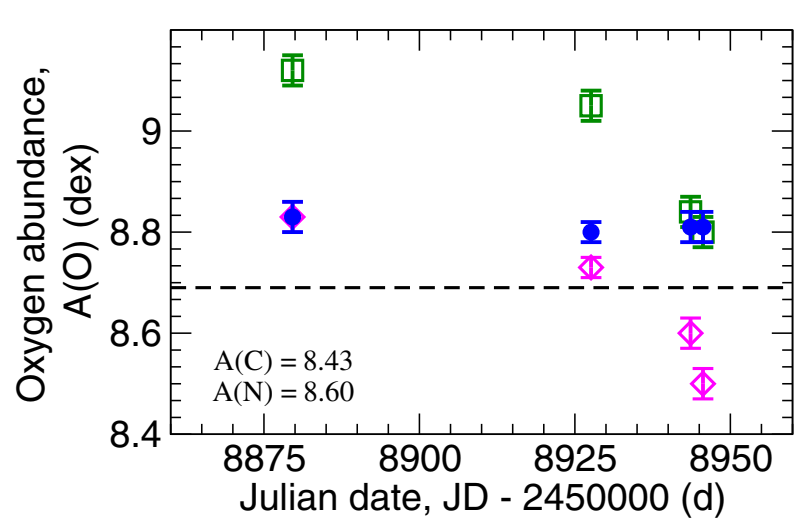

Fig. 3 Conditional chemical abundances of carbon, nitrogen, and oxygen in the atmosphere of Betelgeuse at four different epochs: 31-01-2020, 19-03-2020, 04-04-2020, and 06-04-2020. Chemical abundances of carbon, nitrogen, and oxygen are calculated under three scenarios. First scenario is effective temperature $T_{\text {eff }}=$ var, the chemical abundances are obtained at parameters according to Table 1 (blue filled circles). Second scenario is the chemical abundances are obtained at the assumption of constant effective temperature $T_{\text {eff }}=3646 \mathrm{~K}$ and $\log g=0.2$ (green open squares). Third scenario is the chemical abundances are obtained at the assumption of constant effective temperature $T_{\text {eff }}=3476 \mathrm{~K}$ and $\log g=0.2$ (pink open diamonds). a Carbon abundances calculated under three scenarios. b Nitrogen abundances calculated under three scenarios. c Oxygen abundances calculated under three scenarios. The dashed lines show solar abundances obtained in this study. Error bars represent standard deviation. Source data are provided as a Source Data file.

to 31-01-2020 (Fig. 5a). In Fig. 5b, the comparison of $\mathrm{CN}$ lines observed on 14-02-2012 and 19-03-2020, and the difference between the two epochs is clearly seen. This $\mathrm{CN}$ molecular line is also stronger on 19-03-2020 compared to 04-04-2020 (Fig. 5c). Figure. 5d presents a comparison between the $\mathrm{CN}$ from 2012 and the most 

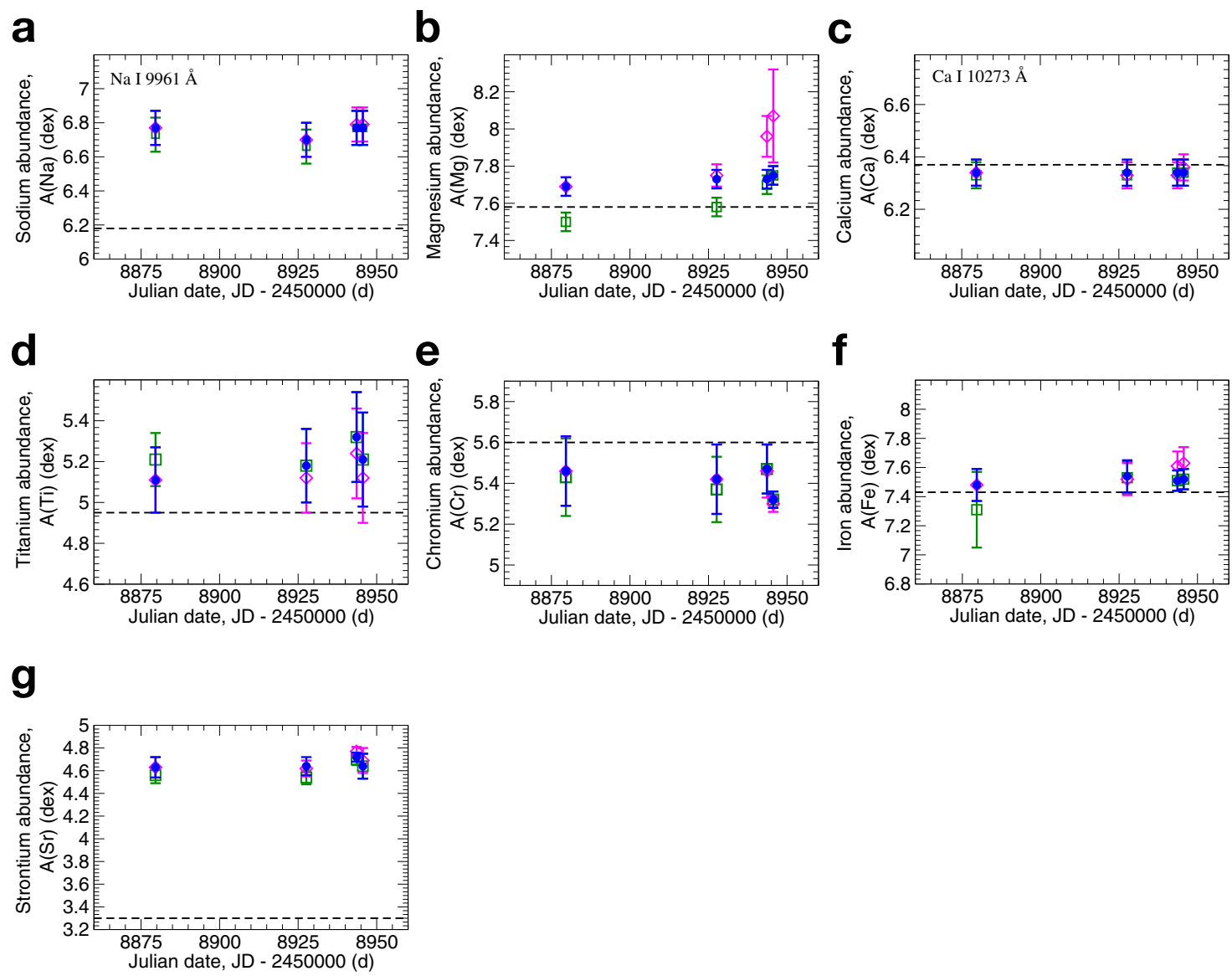

Fig. 4 Chemical composition in the atmosphere of Betelgeuse at four different epochs: 31-01-2020, 19-03-2020, 04-04-2020, and 06-04-2020. a Na abundances calculated under three scenarios. $\mathbf{b} \mathrm{Mg}$ abundances calculated under three scenarios. $\mathbf{c} \mathrm{Ca}$ abundances calculated under three scenarios. $\mathbf{d} \mathrm{Ti}$ abundances were calculated under three scenarios. e $\mathrm{Cr}$ abundances were calculated under three scenarios. $\mathbf{f}$ Fe abundances were calculated under three scenarios. $\mathbf{g}$ Sr abundances calculated under three scenarios. Everywhere, the meanings of symbols are the same as in Fig. 3. The dashed lines show solar abundances obtained in this study. Error bars represent standard deviation. Source data are provided as a Source Data file.

recent spectrum on 06-04-2020. Although the spectral resolution of $14-02-2012$ is slightly higher $(R=65,000)$ than that of other epochs $(R=40,000)$, however, it still allows seeing the significant difference in line strength (Fig. 5a-d). Together with observations as shown by dots, we present the best theoretical fits calculated with parameters specified on each panel according to the obtained values in Table 1. If we assume the constant chemical composition of $\mathrm{C}, \mathrm{N}, \mathrm{O}$ during all epochs, the difference in the strength of line can be interpreted by different temperatures. Everywhere, carbon, nitrogen, and oxygen abundances are assumed to have constant values $8.43,8.6$, and 8.8 dex, respectively.

Comparison with other studies. Levesque and Massey ${ }^{2}$ claimed that the obtained small drop, $\Delta T_{\text {eff }}=50 \mathrm{~K}$, is insufficient to explain Betelgeuse's recent optical dimming and they proposed that episodic mass loss and an increase in the amount of circumstellar dust along the sightline to Betelgeuse is the most likely explanation for its recent photometric evolution. We have obtained different results. For example, at the dimming episode on 31-01-2020, our $T_{\text {eff }}=3476 \pm 4 \mathrm{~K}$ which is by $124 \mathrm{~K}$ lower compared to $T_{\text {eff }}=3600 \pm 25 \mathrm{~K}$, obtained by ref. ${ }^{2}$ on 2020 February 15. Although both studies are based on temperaturesensitive $\mathrm{TiO}$ molecular lines, there are some differences in the methods. Levesque and Massey ${ }^{2}$ used the conventional, welltested methodology, which was presented earlier in refs. ${ }^{9,15}$ and tested on 74 Galactic RSGs. We have found that our technique provides a good agreement with ${ }^{15}$ for 7 common RSGs with a range of effective temperatures from $3440 \mathrm{~K}$ ( $\alpha \mathrm{Her}$ ) to $3815 \mathrm{~K}$ (HD 216946). We conclude that the differences in the methods could not explain the different results for Betelgeuse in the dimming episode.

The ESO-VLT observations ${ }^{1}$ provided the images of the Betegeuse's surface before and after its dimming in January and December 2019. It is clearly seen, that the star has faded, and also its shape was seemingly changing indicating that something happened in the photosphere of the star or in its surroundings. The dark region seen in the optical VLT observations could be explained by a large spot, whose temperature is lower than the typical temperature of Betelgeuse before the dimming. Our findings are in line with these ESO-VLT observations, since the derived temperature drop could be caused by this large spot. We conclude that the radius of the star, $\mathrm{R}$, would not change more than $12 \%$ during the period of brightness recovering by assuming a $3 \sigma$ limit on the change of $\log g=0.12$. This is in line with the ESO-VLT observation ${ }^{1}$, which demonstrated little or no radial change in the star.

The sub-mm observations from the JCMT and APE showed that sub-mm flux was declined by $20 \%$ compared to values before the dimming event ${ }^{7}$. The authors concluded that the dust envelope could not lead to the sub-mm flux reduction and only changes in the photosphere of a star (change in radius or temperature) might be responsible for this reduction. If the radius is supposed to be constant, the temperature drop is $200 \mathrm{~K}$, which could be due to the appearance of the star spots with $T_{\text {eff }}=3250 \mathrm{~K}$ 

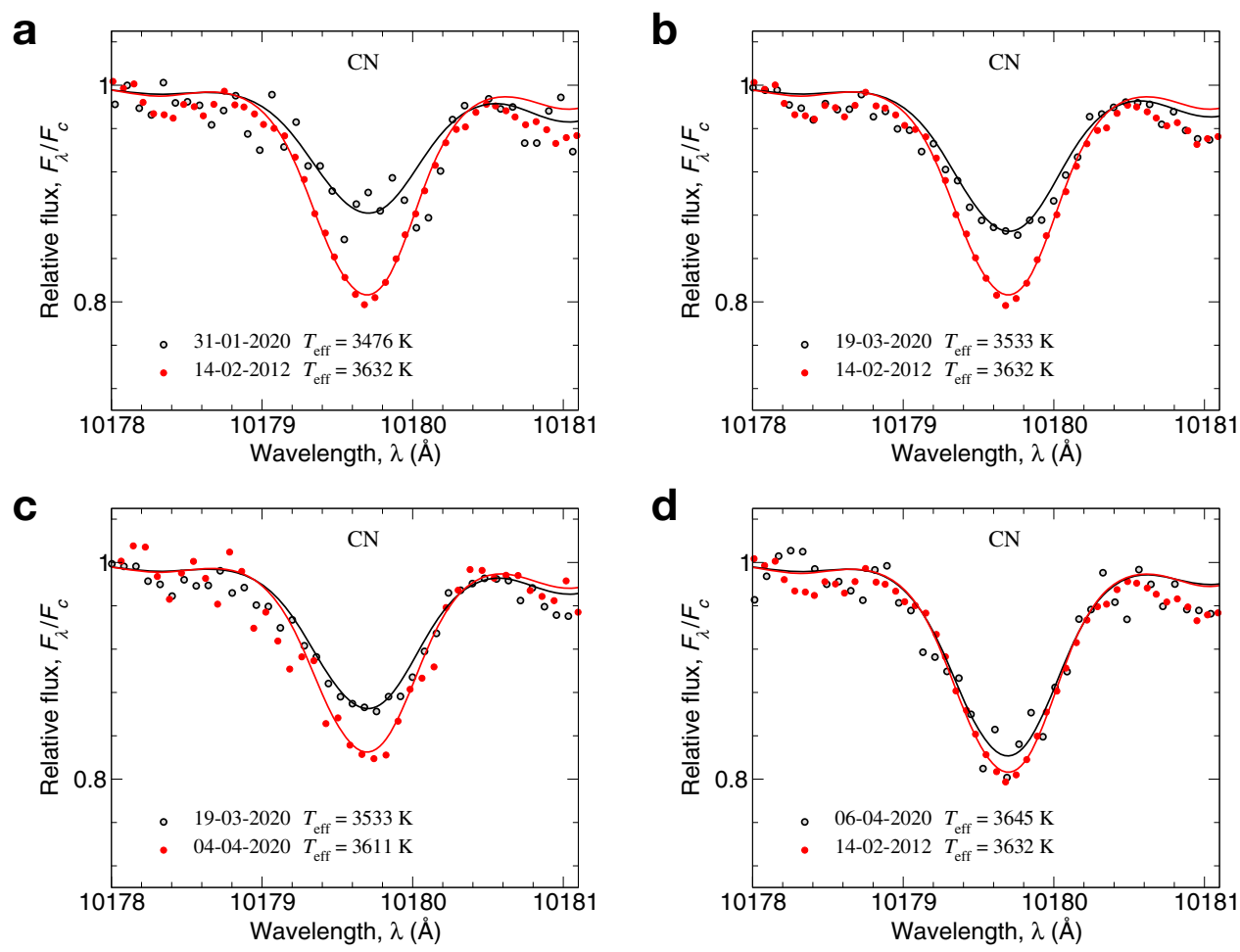

Fig. 5 The different strength of CN (cyanide) molecular line at 10,179 $\AA$ at different epochs can be explained by different temperatures at these epochs. Relative flux is calculated as a ratio of the flux at a particular wavelength $\left(F_{\lambda}\right)$ to the flux in continuum $\left(F_{c}\right)$. The spectral observations are shown by dots. Solid curves are theoretical spectra calculated at the particular temperature specified on each panel, convolved with the instrumental profile. Carbon, nitrogen, and oxygen abundances are 8.43, 8.60, and 8.80 dex, respectively. This line was presented as an example. Similar behavior was found for other CN lines at $\lambda \lambda 10,223,10,367,10,376$, and 10,377 A. a The CN molecular line became stronger on 14-02-2012 compared to 31-01-2020. On the b, the comparison of CN lines observed on 14-02-2012 and 19-03-2020. c This CN molecular line is stronger on 19-03-2020 compared to 04-04-2020, while $\mathrm{CN}$ molecular lines look similar at both epochs 14-02-2012 and 06-04-2020 for which the temperature difference is only $13 \mathrm{~K}$ (d). Source data are provided as a Source Data file.

cover $50 \%$ of the visible surface or spots with $T_{\text {eff }}=3350 \mathrm{~K}$ cover $70 \%$ of the visible surface. These star-spots scenarios would also be accompanied by a $\sim 0.9$ mag dimming in the $V$-band, and are qualitatively similar to the spectrum of Betelgeuse in ref. ${ }^{2}$. We found a similar temperature drop $(170 \mathrm{~K})$ in our study based on high-resolution near-IR observations of Betelgeuse.

The potential changes in the circumstellar flow during the dimming in 2020 February was investigated ${ }^{5}$ by employing SOFIA-EXES high spectral-resolution observations of [Fe II] 25.99 $\mu \mathrm{m}$ and $\left[\mathrm{S}_{\mathrm{I}}\right] 25.25 \mu \mathrm{m}$ emission lines from Betelgeuse. They detected very small changes in the gas velocities in the circumstellar flow, which may indicate the dimming could not be due to dust.

Recently ${ }^{6}$, addressed the $\mathrm{TiO}$ variation based on photometry and did detailed temperature change calculations by comparing to synthetic photometry from the MARCS model photospheres and spectra. In both studies, ref. ${ }^{6}$ and this work, the $\mathrm{TiO}$ molecular absorption lines, which are strongly sensitive to temperature were employed, red/near-IR spectral ranges were investigated, and MARCS model atmospheres were adopted for comparison with theory. They employed photometric observations with three-band wing red/near-IR filters with central wavelengths at $7190,7540,10,240 \AA$, while we used the highresolution spectral analysis at 7000-11000 $\AA$ wavelength range. According to ref. ${ }^{6}, T_{\text {eff }}$ declined from $3645 \pm 15 \mathrm{~K}$ (2019 September 21, 7 days bin) to $3520 \pm 25 \mathrm{~K}$ (2020 February 15, 22, and 29,7 days bins), i.e., $\Delta T_{\text {eff }} \simeq-125 \mathrm{~K}$, that is in line with our result. In Supplementary Fig. 1, we illustrate $T_{\text {eff }}$ data derived from the $\mathrm{TiO}$ index in ref. ${ }^{6}$ and our work. Both studies agree with each other well and provide a full picture of the temperature dropping during the period of the dimming. Since ref. ${ }^{6}$ adopted an oxygen abundance of 0.28 dex lower compared to our study, it might lead to a systematic shift in temperature by $\sim+20-30 \mathrm{~K}$ compared to our $T_{\text {eff }}$ (the higher oxygen abundance in the atmosphere, the stronger molecular $\mathrm{TiO}$ lines employed as temperature indicators).

The detected variations in the $\mathrm{Mg}$ II $\mathrm{k}$-line profiles from the ultraviolet HST/STIS observations suggested movement of material outwards ${ }^{4}$. It was assumed that this outflow of material from the star was due to convective photospheric elements. These convective elements could cause the observed temperature drop, which was presented in ref. ${ }^{7}$ and our study.

\section{Discussion}

During the period from October 2019 to mid-March 2020, Betelgeuse became fainter by more than one magnitude and it is the most significant dimming observed in the recent decades. We were motivated to search for possible explanations of this fading and carried out spectroscopic studies of Betelgeuse at the near-IR spectral region with the high-resolution and high-S/N spectra of Weihai Observatory of Shandong University obtained on 2020 January 31, March 19, April 4 and April 6 and the Observatory of the Canada France Hawaii Telescope (CFHT) obtained on 2012 February 14.

We present a method to deduce $T_{\text {eff }}$ and $\log g$, see "Methods" and the "Effective temperature and surface gravity" subsections. The method is based on two (almost independent) indicators: the 
forest of $2365 \mathrm{TiO}$ lines in the near-IR range at 7700-7900 $\AA$, which is highly sensitive to $T_{\text {eff }}$ and almost not sensitive to the gravity, and the wings of $\mathrm{Ca}$ II lines at 8542 and $8662 \AA$, which are a good indicator for gravity and slightly sensitive to temperature. Usually, the spectrophotometry and low-resolution spectra $(R \sim 1000)$ of broad $\mathrm{TiO}$ bands are preferred, see e.g., ref. ${ }^{16}$. Both methods are based on the TiO lines, which are highly sensitive to temperature, however, instead of low resolution, we analyzed high-resolution $(R \geq 40,000)$ spectra in the near-IR region. We believe that the advantage of our method is the employment of an MCMC forward-modeling for fitting the data, where many different ways of spectral normalization are considered. It avoids the normalization by the eye and helps to find the best normalization.

We show that the effective temperature of Betelgeuse was dropping at least by $170 \mathrm{~K}$ during the period of its great dimming.

From January to April 2020, the effective temperature was recovering to its original value. For example, at post-dimming epoch on 06-04-2020 $T_{\text {eff }}=3646 \pm 9 \mathrm{~K}$, while $T_{\text {eff }}$ was $3632 \pm 7 \mathrm{~K}$ at pre-dimming epoch on 14-02-2012. The rate of recovering correlated with the $V$ magnitude well.

We believe it is unlikely that the whole star became cooler by $170 \mathrm{~K}$ and attribute it to the emergence of a dark large spot on the surface on Betelgeuse. If we assume that the dark spot covers $50 \%$ of the visible surface of the star on 31-01-2020, the temperature of the dark spot could be $340 \mathrm{~K}$ lower than the usual temperature $\left(T_{\text {eff }}=3646 \mathrm{~K}\right)$. A similar suggestion was also presented by ${ }^{7}$, who found a similar temperature drop $200 \mathrm{~K}$ in the minimum of brightness and reconciled it by star spots.

We did not find any significant changes in surface gravity at all epochs of observations from 31-01-2020 to 06-04-2020 and $\log g$ keeps their constant values within the error bars with a mean value $\log g=0.22 \pm 0.04$. According to $g=\mathrm{GM} / R^{2}$, and taking a $3 \sigma$ limit on the change of $\log g=3 * 0.04=0.12$, we conclude that the radius of the star was constant within $12 \%$ during the period of brightness recovering from 31-01-2020 to 06-04-2020.

Using two independent indicators to estimate $T_{\text {eff }}$ and $\log g$, we rule out pulsation as a possible mechanism of temperature decreasing. Since we know that $T_{\text {eff }}$ was changing by $170 \mathrm{~K}$ (independent of $\log g$ ) and it is enough to explain the change in $V \sim 0.918$, it is unlikely that the radius of the star was changing, according to $L \sim T_{\text {eff }}^{4} R^{2}$. Otherwise, even a small change in radius would lead to a change in luminosity and we would have much deeper dimming (if $T_{\text {eff }}$ decreases by $170 \mathrm{~K}$ and $R$ decreases simultaneously), no change in brightness (if $T_{\text {eff }}$ decreases by 170 $\mathrm{K}$ and $R$ increases by $10 \%$ ), or even brightening of the star (if $T_{\text {eff }}$ decreases by $170 \mathrm{~K}$ and $R$ increases by more than $10 \%$ simultaneously).

We obtained the chemical compositions of Betelgeuse at the pre-dimming, dimming, and post-dimming episodes based on near-IR spectral region, where normalization can be done reliably (Supplementary Table 2). The developed line list was tested with the solar spectrum. The abundances for $\mathrm{C}, \mathrm{N}, \mathrm{O}, \mathrm{Na}, \mathrm{Mg}, \mathrm{Ca}, \mathrm{Ti}$, $\mathrm{Cr}, \mathrm{Fe}$, and $\mathrm{Sr}$ would remain constant during the dimming episode if the variable temperature is considered. In contrast, if we assume a constant temperature (the highest $T_{\text {eff }}=3646 \mathrm{~K}$ or the lowest one $3476 \mathrm{~K}$ ) at the period of changing magnitude, we would face difficulty in explaining the changing of these chemical abundances in a short period of time.

\section{Methods}

Observations and data reduction. The observation list of Betelgeuse is presented in Supplementary Table 3. High-resolution $(R \sim 40000)$ Echelle spectra of Betelgeuse with a wavelength range of 3710-11,000 A were obtained on 2020 January 31, March 19, April 4, and April 6 with the bench-mounted, stabilized, fiber-fed Weihai Echelle Spectrograph (WES) ${ }^{29}$ attached to the $1 \mathrm{~m}$ telescope at the Weihai Observatory of Shandong University ${ }^{30}$, located at $122^{\circ} 02^{\prime} 58.6 \mathrm{E}$ and $37^{\circ} 32^{\prime} 09.3 \mathrm{~N}$.
The telescope has an f/8 classic Cassegerain design and equatorial fork mount mechanical structure. The spectrograph covers 3710 to $11,000 \AA$ in 107 spectral orders over the rectangular CCD. The exposure time $\left(t_{\text {exp }}\right)$ was $1200 \mathrm{~s}$ on January 31 and March 19 and $480 \mathrm{~s}$ on April 4 and 6 due to the increase of brightness. The $\mathrm{S} / \mathrm{N}$ ratio measured at the continuum near $9000 \AA$ ranges from 80 in April to 500 on March 19. The reduction of the CCD images was carried out in the context of the Image Reduction and Analysis Facility IRAF (IRAF is distributed by the National Optical Astronomy Observatories, which are operated by the Association of Universities for Research in Astronomy under a cooperative agreement with the National Science Foundation), including bias subtraction, flat fielding, background subtraction, wavelength calibration, continuum normalization, and flux calibration. The observations cover the epoch of the dimming and the subsequent recovery of brightness. On 2020 January 31, the $V$ magnitude was 1.609 mag, then Betelgeuse became slightly brighter with $V=1.144$ mag on March 19, and further recovery with $V=0.754 \mathrm{mag}$ and $V=0.691 \mathrm{mag}$ on April 4, and 6, respectively. Each $V$ magnitude was calculated by averaging over all available magnitudes in the American Association of Variable Star Observers (AAVSO) database in a particular date of observations. The error for each $V$ magnitude is a standard deviation.

We used the high-resolution $(R=65000)$ spectra of Betelgeuse from the visible to the near-IR ranges obtained on 2012 February 14 with the Echelle Spectro Polarimetric Device for the Observation of Stars (ESPaDOnS) ${ }^{31}$ attached to the $3.6 \mathrm{~m}$ telescope of the CFHT Observatory located on the summit of Mauna Kea, Hawaii [http://www.cfht.hawaii.edu/Instruments/Spectroscopy/Espadons/]. Observations with this spectrograph cover the wavelength region from 3690 to $10,480 \AA$ and exposure time $0.8 \mathrm{~s}$. Calibrated intensity spectral data were extracted from the ESPaDOnS archive through the Canadian Astronomical Data Centre (CADC). The $\mathrm{S} / \mathrm{N}$ measured at the continuum near $6000 \AA$ was estimated as 800 for Betelgeuse.

Model atmospheres and spectrum calculations. Spherical local thermodynamic equilibrium (LTE) model atmospheres MARCS $^{32}$ were used in our calculations. Extensive line lists for M-type stars were extracted via the facilities of the VALD3 [http://vald.astro.uu.se/ vald3/php/vald.php] database $\mathrm{e}^{33}$. The synthetic line profiles are calculated by the code SYNTHV_NLTE ${ }^{34}$. The departure coefficients, $b_{i}=$ $n_{\mathrm{NLTE}} / n_{\mathrm{LTE}}$, were computed with the code DETAIL ${ }^{35}$ based on the method of accelerated $\Lambda$ iteration ${ }^{36}$. Here, $n_{\text {NLTE }}$ and $n_{\text {LTE }}$ are the statistical equilibrium and thermal (Saha Boltzmann) number densities, respectively. The BINMAG v.6 code [http://www.astro.uu.se/ oleg/binmag.html] ${ }^{37}$ was used for automatic spectral line fitting and comparison with the observed spectrum. Throughout this study, the element abundances are determined from line profile fitting.

The Sun was adopted as the benchmark star for testing all lines in the near-IR region. We used the MARCS model atmosphere 5777/4.44/0 and a depthindependent microturbulence of $0.9 \mathrm{~km} \mathrm{~s}^{-1}$. The solar flux observations were taken from the Kitt Peak Solar Atlas ${ }^{38}$. Our synthetic flux profiles were convolved with a profile that combines a rotational broadening of $1.8 \mathrm{~km} \mathrm{~s}^{-1}$ and broadening by macroturbulence with a radial-tangential profile. For different lines, the most probable macroturbulence velocity, $V_{\text {mac }}$, was varied between 2 and $4 \mathrm{~km} \mathrm{~s}^{-1}$. Hereafter, the element abundances are given in the scale, where for hydrogen $\mathrm{A}$ $(\mathrm{H})=12$.

Effective temperature. Typically, the low resolution $(R \sim 1000)$ spectrophotometry is used to determine the effective temperatures of RSGs. The absolute line strengths of $\mathrm{TiO}$ features at $\lambda \lambda 4761,4954,5167,5448,5847,6158,6658$, and $7054 \AA$ are measured and they allow us to avoid the spectral normalization procedure. The same $\mathrm{TiO}$ features are also employed to define the spectral types of $\mathrm{M}$ stars, e.g., see ref. ${ }^{16}$.

Here, for temperature determination, we have developed an alternative method based on a forest of $\mathrm{TiO}$ lines in the near-IR range at 7700-7900 A. Totally, 2365 $\mathrm{TiO}$ lines can be found in this range. The forest of molecular TiO lines in 7700-7900 $\AA$ is highly sensitive to temperature and only slightly to gravity. We set up the $\log g$ to be constant at all epochs of observations and equal to 0.0 . The theoretical spectra in the region of $7700-7900 \AA$ were calculated with $V_{\text {mic }}=$ $3 \mathrm{~km} \mathrm{~s}^{-1}, V \sin i=5 \mathrm{~km} \mathrm{~s}^{-1}, V_{\text {mac }}=16 \mathrm{~km} \mathrm{~s}^{-1}$, and the carbon, nitrogen, oxygen, calcium, and titanium abundances are $\mathrm{A}(\mathrm{C})=8.43, \mathrm{~A}(\mathrm{~N})=8.6, \mathrm{~A}(\mathrm{O})=8.8^{39}, \mathrm{~A}$ $(\mathrm{Ca})=6.34$, and $\mathrm{A}(\mathrm{Ti})=4.95$. The dissociation energy of $D_{0}(\mathrm{TiO})=6.87 \mathrm{eV}^{40}$. The convolution with an instrumental profile was done according to the spectral resolution in Supplementary Table 3. The model spectra were calculated with a step of $50 \mathrm{~K}$ for temperature, within this range, the linear interpolation was applied.

Since the lines are blended with each other, it complicates the finding of the exact level of continuum. The problem of the spectral normalization procedure was solved by including the coefficient of re-normalization (Norm) as a variable parameter in Monte Carlo simulations. It allows simulating many different ways of normalization and its impact on the obtained temperature. Although, Norm does not play any physical role and was only considered for the accuracy of $T_{\text {eff }}$ and $\log g$ determination.

We fit the forest of TiO lines in 7700-7900 $\AA$ to infer the effective temperature using an MCMC forward-modeling method built on the EMCEE package ${ }^{41}$. The uncertainty is taken as the difference between the 84th and 50th percentile as the upper limit, and the difference between the 50th and 16th percentile as the lower limit for all model parameters. If the posterior distributions follow normal 

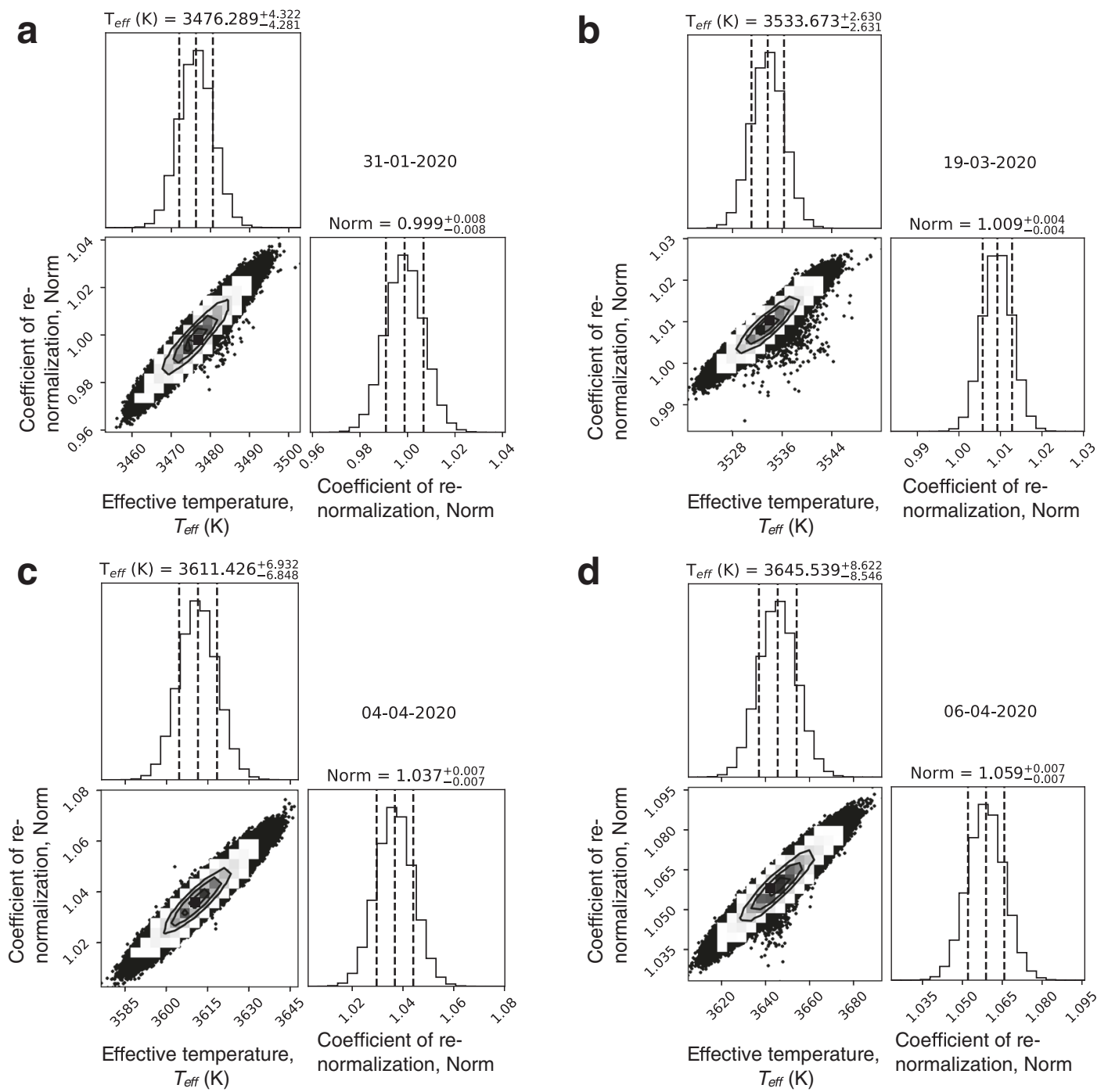

Fig. 6 Four corner plots with the final effective temperatures $\left(T_{\text {eff }}\right)$ of Betelgeuse obtained at four epochs 31-01-2020, 19-03-2020, 04-04-2020, and 06-04-2020. a The best-fit $T_{\text {eff }}$ and its uncertainties were obtained on 31-01-2020. The panel corresponding to the fit of the region at 7700-7900 $\AA$ is shown in Supplementary Fig. 4a. $\mathbf{b}$ The best-fit $T_{\text {eff }}$ and its uncertainties were obtained on 19-03-2020. The panel corresponding to the fit of the region at 7700-7900 $\AA$ is shown in Supplementary Fig. 4b. c The best-fit $T_{\text {eff }}$ and its uncertainties were obtained on 04-04-2020. The panel corresponding to the fit of the region at 7700-7900 $\AA$ is shown in Supplementary Fig. 4c. d The best-fit $T_{\text {eff }}$ and its uncertainties were obtained on 06-04-2020. The panel corresponding to the fit of the region at 7700-7900 $\AA$ is shown in Supplementary Fig. 4d. On each panel, the diagonal shows the marginalized posteriors. The subsequent covariances between all the parameters are in the corresponding 2D histograms. The vertical lines represent the 16, 50, and 84 percentiles. The best-fit parameters for $T_{\text {eff }}$ and their uncertainties are presented on the top of each panel. Uncertainty for $T_{\text {eff }}$ is defined as the difference between the 84th and 50th percentile as the upper limit, and the difference between the 50th and 16th percentile as the lower limit. The data of observations are marked in dd-mm-yyyy format.

(Gaussian) distributions then this equates to the $1 \sigma$ uncertainty for each parameter. The corner plots ( $T_{\text {eff }}$ vs. Norm) at four epochs of observations on 31-01-2020, 1903-2020, 04-04-2020, and 06-04-2020 are presented in Fig. 6 and in Supplementary Fig. 2a at 14-02-2012. The best fits of TiO bands for all investigated epochs of observations are presented in Fig. 7, where the $2365 \mathrm{TiO}$ lines are marked by vertical lines on panel a.

Surface gravity. To estimate the surface gravity, we used the same method as for the effective temperature. However, instead of the forest of TiO lines, we adopted the wings of Ca II lines at 8542 and $8662 \AA$, which are a good indicator for gravity and almost not sensitive to temperature. The model spectra were calculated with the determined $T_{\text {eff }}$ for each epoch of observations. We take a step of $\log g$ of 0.2 dex, and within this range, linear interpolation was applied. The Ca II lines at 8542 and $8662 \AA$ are not a subject of the non-local thermodynamic equilibrium (NLTE) effects (Sitnova, priv. comm.) and can be used in the LTE assumption. The corner plots ( $\log g$ vs. Norm) at four epochs of observations on 31-01-2020, 19-03-2020,
04-04-2020, and 06-04-2020 are presented in Supplementary Fig. 3, and at 14-022012, in Supplementary Fig. 2 b.

Since the Ca II lines at 8542 and $8662 \AA$ are blended with plenty of other lines, we created a mask, which consists of some parts of the spectrum. These parts are presented in Supplementary Fig. 4a, b for two epochs of observations: panel a presents Ca II lines at the epoch of 14-02-2012 and panel b presents Ca II lines at 8542 and $8662 \AA$ at the epoch of 31-01-2020. Only these spectral parts were taken for fitting, the result of which is presented by corner plots Supplementary Figs. $2 \mathrm{~b}$ and 3. In Supplementary Fig. 4c, we demonstrate the fitting of the wings of Ca II lines at 8542 and $8662 \AA$ in the solar spectrum with the model ones. It allows to check atomic data such as van der Waals broadening constants, oscillator strengths to be sure that we are able to fit the wings of Ca II lines at 8542 and $8662 \AA$ properly.

Chemical composition. The advantage of this research is that we used spectral observations at the near-IR region (8000-11,000 $\AA$ ) to derive the chemical composition. The spectral continuum can be determined reliably at this wavelength 
a

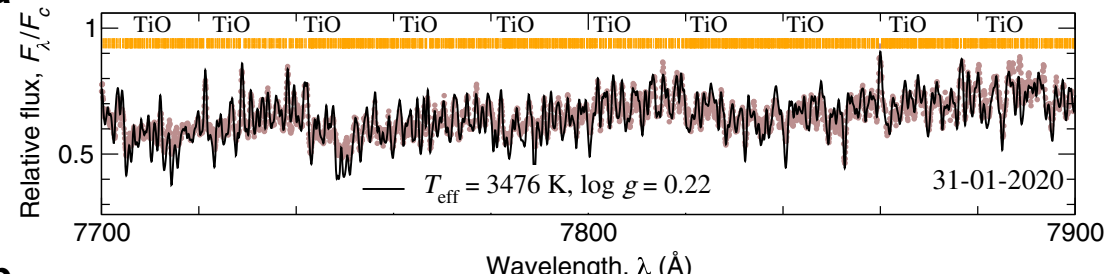

b

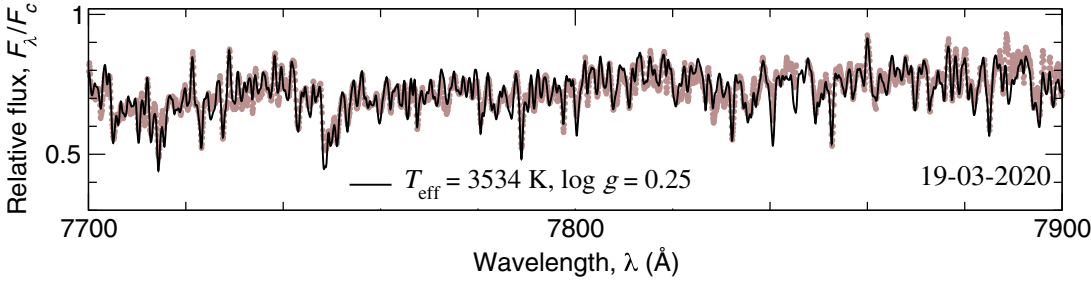

C
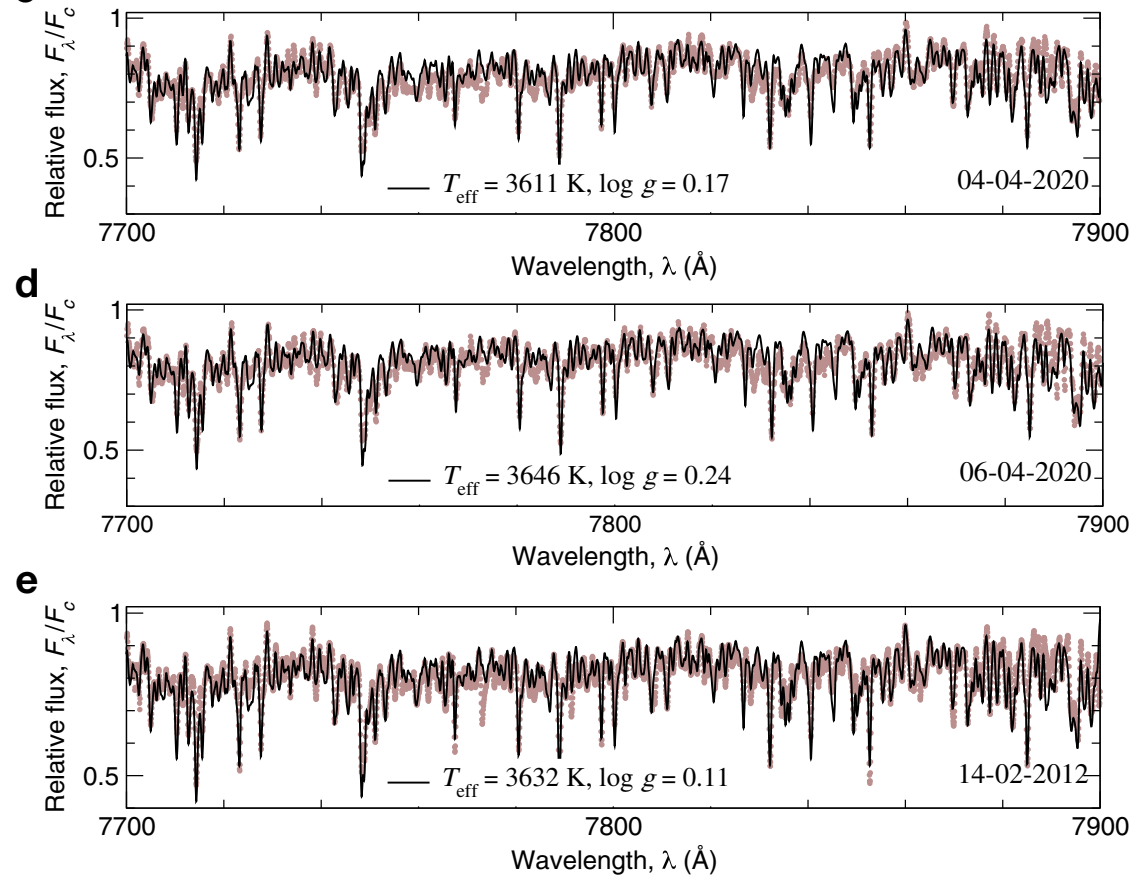

Fig. 7 Best fits to the molecular bands of TiO for five sets of spectra. The observed spectra of Betelgeuse obtained on 31-01-2020 (a), 19-03-2020 (b), 04-04-2020 (c), 06-04-2020 (d), and 14-02-2012 (e) are shown by brown dots. The solid line is a theoretical profile calculated with the particular effective temperature $\left(T_{\text {eff }}\right)$ and surface gravity $(\log g)$, which were obtained in this study and presented on each panel. Relative flux is calculated as a ratio of the flux at a particular wavelength $\left(F_{\lambda}\right)$ to the flux in continuum $\left(F_{c}\right)$. The 2365 TiO lines are marked by vertical lines on the $\mathbf{a}$. Source data are provided as a Source Data file.

range. Nevertheless, for example, in the blue region (4000-6000 $\mathrm{A})$, it is hard to find the location of continuum level, since there are plenty of atomic lines and molecular bands blended with each other. The chemical composition was derived in the LTE assumption except for $\mathrm{Na}$ and $\mathrm{Mg}$, for which the deviations from LTE were considered. The line list was developed according to the near-IR spectral region and in the first place, it was tested on the solar spectrum before applying to the Betelgeuse. Using this line list, we obtained chemical abundances from the Sun and then compared it with Betelgeuse.

The microturbulent velocity, $V_{\text {mic }}=3 \mathrm{~km} \mathrm{~s}^{-1}$, was obtained from Fe I lines listed in Supplementary Table 4 . The synthetic profiles were convolved with a profile that combines a rotational broadening of $5 \mathrm{~km} \mathrm{~s}^{-1}$ and broadening by macroturbulence with a radial-tangential profile. Macroturbulence is introduced to reproduce the line profile shape, and it does not affect the derived element abundance, if the line profile is fitted well. For different lines of $\mathrm{Fe}$ I, the macroturbulence velocity, $V_{\text {mac }}$, was varied between 10 and $15 \mathrm{~km} \mathrm{~s}^{-1}$. Supplementary Fig. $4 \mathrm{~d}$ demonstrates the iron abundances vs. the excitation energy $\left(E_{\text {exc }}\right)$ of the lower level for the investigated $\mathrm{Fe}_{\mathrm{I}}$ lines for two epochs of observations: 14-02-2012 and 31-01-2020. In both epochs, there is no trend for A $(\mathrm{Fe})$ vs. $E_{\mathrm{exc}}$ and the mean values of iron abundances are $\mathrm{A}(\mathrm{Fe})=7.51 \pm 0.11$ and $\mathrm{A}$ $(\mathrm{Fe})=7.53 \pm 0.15$. Iron abundances from individual lines at all epochs of observations are presented in Supplementary Table 5. Using the same line list, we obtained $\mathrm{A}(\mathrm{Fe})=7.41 \pm 0.09$ for the Sun.
Below, we briefly describe the method of chemical abundance determination for a particular element.

To determine $\mathrm{C}, \mathrm{N}, \mathrm{O}$ abundances, we employed $\mathrm{CN}$ molecular lines presented in Supplementary Table 1. The best fits of CN molecular line 10,179 $\AA$ are presented in Fig. 5. The dissociation energy is $D_{0}(\mathrm{CN})=7.65 \mathrm{eV}^{42}$. The $\mathrm{CN}$ lines depend not only on $\mathrm{C}$ and $\mathrm{N}$ abundances, but also on $\mathrm{O}$ abundance in the atmosphere $^{39}$. As a result, all presented C, N, O abundances are conditional (Fig. 3). For example, when we measure $C$, we keep $A(N)=8.6$ and $A(O)=8.8$. When we measure $N$, we keep $\mathrm{A}(\mathrm{C})=8.43$ and $\mathrm{A}(\mathrm{O})=8.8$. Since, we were not able to get independent values of $\mathrm{C}, \mathrm{N}$ and $\mathrm{O}$ abundances, we adopted the initial values of them from ${ }^{39}$. We have found a set of values of carbon, nitrogen, and oxygen abundances $\mathrm{A}(\mathrm{C})=8.43, \mathrm{~A}(\mathrm{~N})=8.6, \mathrm{~A}(\mathrm{O})=8.8$, at which we can get a good agreement between observational $\mathrm{CN}$ molecular lines and theoretical profiles.

We employed only one line of Na I at $9961 \AA$. The NLTE calculations with the model atom adapted from ref. ${ }^{43}$ showed that LTE abundances can be overestimated by $\sim 0.20$ dex in the atmosphere with $T_{\text {eff }}$ from 3476 to $3646 \mathrm{~K}$ and $\log g=0.2$. However, in the solar atmosphere, the NLTE correction is only 0.02 dex for $\mathrm{Na}$ I line at $9961 \AA$. Since we employed only one line, the error bars were estimated from uncertainties in continuum level by $1 \%$. We obtained that $\mathrm{Na}$ abundance in the atmosphere of Betelgeuse is much higher by about $0.60 \mathrm{dex}$ relative to solar value, where the solar value was obtained from the same line in the solar spectrum. 
Table 2 Physical parameters of red supergiants obtained in this study.

\begin{tabular}{|c|c|c|c|c|c|c|c|c|c|c|}
\hline Star & Name & $T_{\text {eff }}(K)$ & $-\sigma\left(T_{\text {eff }}\right)$ & $+\sigma\left(\boldsymbol{T}_{\mathrm{eff}}\right)$ & $\log g$ & $-\sigma(\log g)$ & $+\sigma(\log g)$ & {$[\mathrm{Fe} / \mathrm{H}]$} & $\sigma([\mathrm{Fe} / \mathrm{H}])$ & $V_{\text {mic }}\left(\mathrm{km} \mathrm{s}^{-1}\right)$ \\
\hline HD 36389 & 119 Tau & 3610 & -8 & +8 & 0.02 & -0.01 & +0.02 & 0.03 & 0.09 & 2.5 \\
\hline HD 44537 & psi01 Aur & 3723 & -9 & +9 & -0.16 & -0.08 & +0.08 & 0.07 & 0.10 & 3 \\
\hline HD 49331 & - & 3691 & -5 & +4 & 0.41 & -0.02 & +0.02 & -0.23 & 0.12 & 3 \\
\hline HD 50877 & omi01 CMa & 4095 & -20 & +21 & -0.15 & -0.10 & +0.08 & -0.02 & 0.19 & 3 \\
\hline HD 52877 & $\sigma \mathrm{CMa}$ & 3735 & -4 & +4 & 0.22 & -0.08 & +0.08 & 0.06 & 0.12 & 2 \\
\hline HD 146051 & $\delta$ Oph & 3729 & -8 & +8 & 0.98 & -0.02 & +0.01 & -0.38 & 0.05 & 2 \\
\hline HD 156014 & $\alpha \mathrm{Her}$ & 3440 & -5 & +5 & 3.08 & -0.15 & +0.15 & 0.59 & 0.06 & 3 \\
\hline HD 165674 & VX Sgr & 3533 & -10 & +11 & 1.98 & -0.03 & +0.02 & 0.61 & 0.45 & 3 \\
\hline HD 192909 & 32 Cyg & 3771 & -8 & +8 & 0.45 & -0.02 & +0.02 & -0.03 & 0.11 & 2 \\
\hline HD 200905 & $\xi$ Cyg & 3964 & -14 & +14 & 0.76 & -0.02 & +0.02 & -0.01 & 0.15 & 2 \\
\hline HD 206936 & $\mu \mathrm{Cep}$ & 3683 & -9 & +9 & -0.26 & -0.08 & +0.08 & 0.26 & 0.25 & 3 \\
\hline HD 208816 & VV Cep & 3662 & -10 & +10 & 0.09 & -0.02 & +0.02 & 0.10 & 0.04 & 3 \\
\hline HD 210745 & $\zeta$ Cep & 4139 & -112 & +77 & 0.59 & -0.03 & +0.03 & 0.22 & 0.18 & 2 \\
\hline HD 216946 & V424 Lac & 3815 & -74 & +93 & 0.49 & -0.03 & +0.03 & 0.21 & 0.10 & 2 \\
\hline HD 217906 & $\beta \mathrm{Peg}$ & 3621 & -5 & +5 & 1.15 & -0.05 & +0.05 & -0.22 & 0.04 & 2 \\
\hline
\end{tabular}

Effective temperature $\left(T_{\text {eff }}\right)$, surface gravity $(\log g)$, metallicity $([\mathrm{Fe} / \mathrm{H}])$, and microturbulent velocity $\left(V_{\text {mic }}\right)$ are given for each star. Uncertainties for $T_{\text {eff }}\left(-\sigma\left(T_{\text {eff }}\right)\right.$ and $\left.+\sigma\left(T_{\text {eff }}\right)\right)$ and log $g(-\sigma(\log g)$ and $+\sigma(\log g))$ are defined as the difference between the 84th and 50th percentile as the upper limit, and the difference between the 50th and 16th percentile as the lower limit. Uncertainties for [Fe/H] $(\sigma$ $([\mathrm{Fe} / \mathrm{H}]))$ are calculated as standard deviation. Source data are provided as a Source Data file.

\section{a}

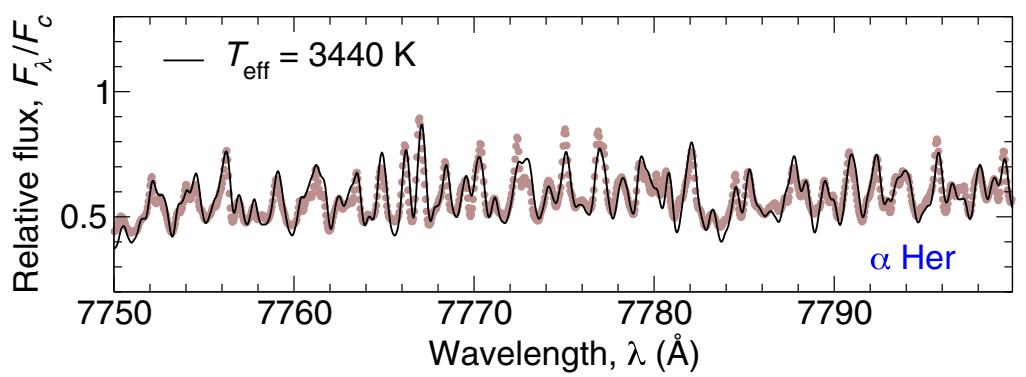

b

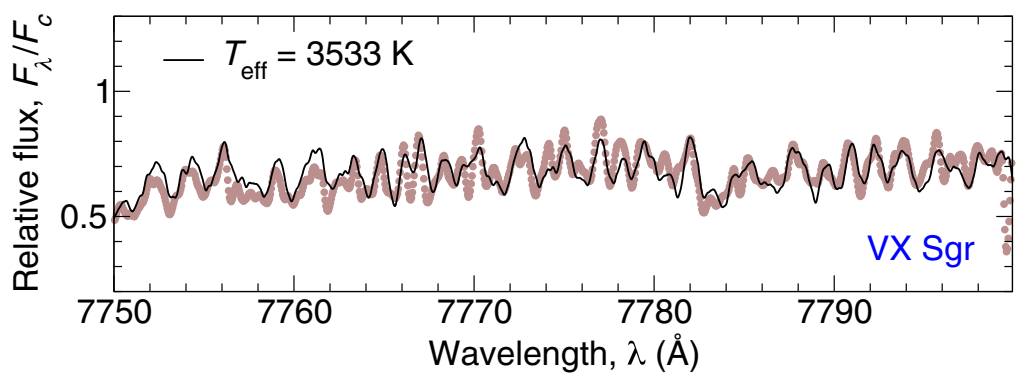

C

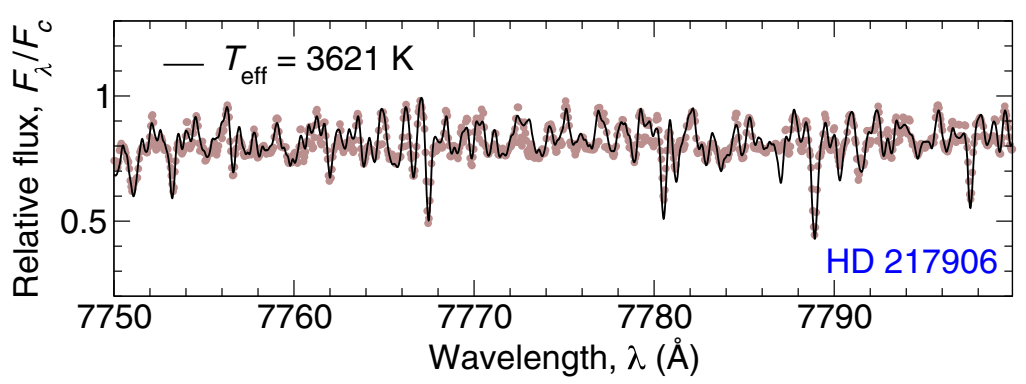

d

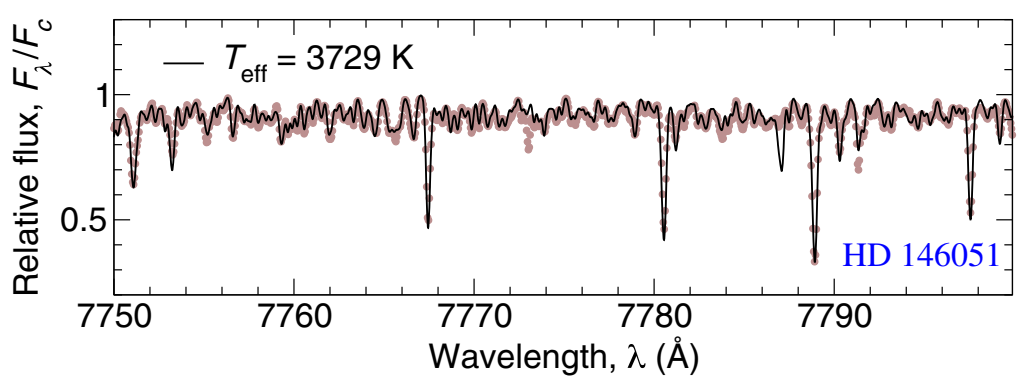

Fig. 8 The best fits of the parts of spectra of Betelgeuse in 7750-7800 A range for four represented supergiant stars. Relative flux is calculated as a ratio of the flux at a particular wavelength $\left(F_{\lambda}\right)$ to the flux in continuum $\left(F_{\mathrm{c}}\right)$. The solid black line is a theoretical profile calculated with the particular $T_{\text {eff }}$ obtained in this study. The observed spectra are shown by brown dots. The name of the star and its $T_{\text {eff }}$ are presented on each panel. a The best-fit for the coolest star in our sample, $\alpha$ Her, with effective temperature $\left(T_{\text {eff }}\right) 3440 \mathrm{~K}$. b The best-fit for VX Sgr with $T_{\text {eff }}=3533 \mathrm{~K}$. c The best-fit for HD 217906 with $T_{\text {eff }}=3621 \mathrm{~K}$. d The best-fit for HD 146051 with $T_{\text {eff }}=3729 \mathrm{~K}$. Source data are provided as a Source Data file. 
We used the wings of the strong $\mathrm{Mg}$ I line at $8806.75 \AA$ and the line at 10,312.53 $\AA$. Although we provide NLTE values for Mg, our NLTE calculations with the model atom adapted from ref. ${ }^{44}$ showed very small NLTE effects in Mg I line at 10,312.53 and the wings of $\mathrm{Mg}_{\mathrm{I}}$ line at $8806.75 \AA$ are not suffering from NLTE at all. We have found slightly overabundance $([\mathrm{Mg} / \mathrm{H}] \sim 0.11)$ of $\mathrm{Mg}$ relative to solar value at all epochs (Fig. 4b).

By employing two $\mathrm{Ca}$ I lines at 6471.66 and $10,273.68 \AA$, we have found that $\mathrm{Ca}$ abundances are close to solar values within the error bars at all epochs of observations.

We measured five lines of $\mathrm{Ti}$ at $\lambda \lambda 10,034.49,10,048.82,10,066.51,10,189.14$, $10,496.11 \AA$, and an unusual big scatter in abundances were noticed. Together with $\mathrm{Fe}$ and $\mathrm{Mg}$, Ti overabundance was found in the atmosphere of Betelgeuse as well.

The similar big scatter was noticed in $\mathrm{Cr}$ abundances, when we employed six $\mathrm{Cr}$ I lines at $\lambda \lambda$ 8707.402, 8707.965, 9016.98, 9035.85, 10,486.25, 10,510.01.

However, using the same line list, the solar $\mathrm{Cr}$ abundance was obtained $\mathrm{A}(\mathrm{Cr})=$ $5.60 \pm 0.13$ with similar big scatter. In contrast to $\mathrm{Fe}, \mathrm{Mg}$, and $\mathrm{Ti}$, the mean values of Chromium in the atmosphere of Betelgeuse are found to be lower compared to solar $\mathrm{Cr}$ abundance.

To measure Sr abundance, we used two strong Sr II lines at 10,036.65 $\AA$ and $10,327.31 \AA$. Solar Sr abundance from these lines is $\mathrm{A}(\mathrm{Sr})=3.3 \pm 0.07$, while in the atmosphere of Betelgeuse, Sr overabundance reaches up to $[\mathrm{Sr} / \mathrm{H}] \sim 1.4$ dex.

Approbation of the method. Following the standard scientific process, we have tested the method with additional 15 red supergiant stars of K- and M- spectral types by employing their high-resolution spectra in the near-IR ranges. The characteristics of the observed spectra are presented in Supplementary Table 6, where $V$ magnitudes were adopted from the SIMBAD database, and S/N was measured on $8000 \AA$. The spectral types of HD $36389, \alpha$ Her, HD 44537, HD 50877, HD 52877, HD 200905, HD 206936, HD 210745, and HD 216946 were adopted from ref. ${ }^{15}$, and from the SIMBAD database for the remaining stars. The spectrum of HD 49331 was extracted from the ESO archive [http://www.eso. org/sci/observing/tools/uvespop.html]. For the remaining stars, their spectra have the same characteristics as the spectrum of Betelgeuse obtained on 2012 February 14.

The physical parameters $\left(T_{\text {eff }} \log g,[\mathrm{Fe} / \mathrm{H}]\right.$, and $\left.V_{\text {mic }}\right)$ derived in this study for 15 additional stars are presented in Table 2 . The obtained effective temperatures are ranged from 3440 to $4139 \mathrm{~K}$, and $\log g$, from -0.26 to +3.08 .

The theoretical spectra were calculated with oxygen and titanium abundances, $\mathrm{A}(\mathrm{O})=8.69$ and $\mathrm{A}(\mathrm{Ti})=4.95$. It should be noted that the values of oxygen and titanium abundances may affect the absolute values of $T_{\text {eff, }}$ which is determined on the base of $\mathrm{TiO}$ molecular lines. Although, we would not expect a deviation of $\mathrm{Ti}$ abundance from the solar value in the atmosphere of RSGs stars, while oxygen composition may deviate from the solar value due to the more advanced evolutionary stage of RSGs. For example, if the oxygen abundance is higher by 0.1 dex, the systematic shift $\sim+40 \mathrm{~K}$ should be considered for the effective temperature. Other parameters, $\log g,[\mathrm{Fe} / \mathrm{H}]$, and $V_{\text {mic }}$, were taken in accordance with Table 2. The theoretical spectra were convolved with a profile that combines a rotational broadening, broadening by macroturbulence, and instrumental profile, $R=65,000$. The macroturbulence was varied between 8 to $16 \mathrm{~km} \mathrm{~s}^{-1}$ for different stars. The quality of the fits is illustrated in Fig. 8 for four representing stars: $\alpha$ Her, VX Sgr, HD 217906, and HD 146051 with the effective temperatures in the range from 3440 to $3729 \mathrm{~K}$.

The comparison with previous studies is presented in Supplementary Fig. 5. For $T_{\text {eff }}<3800 \mathrm{~K}$, our $T_{\text {eff }}$ are in good agreement with data from ref. ${ }^{15}$, where $T_{\text {eff }}$ were derived from the MARCS models using broad-band photometry. For three stars with $T_{\text {eff }}$ from $\sim 3800$ to $\sim 4100 \mathrm{~K}$, our temperatures are higher by about $\sim 150 \mathrm{~K}$ compared to ${ }^{15}$ (Supplementary Fig. 5a).

For nine common stars, our effective temperatures are in good agreement with ref. ${ }^{45}$, whose temperatures were obtained by comparing BT-SETTL model atmospheres to spectral energy distributions (SEDs) created from Hipparcos data. Although, there are three outliers. For VX Sgr, ref. ${ }^{45}$ have obtained lowtemperature $T_{\text {eff }}=2255 \mathrm{~K}$, while our result is $T_{\text {eff }}=3533 \mathrm{~K}$. This star was not included in Supplementary Fig. 5b. The other two outliers are HD 52877 and HD 206936. For the first of them, our $T_{\text {eff }}$ is lower by $\sim 200 \mathrm{~K}$ compared to ref. ${ }^{45}$, while for the second of them our $T_{\text {eff }}$ is higher by $\sim 300 \mathrm{~K}$ compared to ref. 45 .

We found six common stars with ref. ${ }^{46}$, who presented the stellar atmospheric parameters by employing the ULYSS program with the ELODIE spectral library. For three stars with $T_{\text {eff }} \leq 3800 \mathrm{~K}$, our temperatures are in line with ref. ${ }^{46}$, however, they are lower by $\sim 200 \mathrm{~K}$ for another 2 stars. One of the outliers is $32 \mathrm{Cyg}$ (HD 192909). We obtained $T_{\text {eff }}=3771 \mathrm{~K}$, which is almost in the middle between $T_{\text {eff }}=$ $3543 \mathrm{~K}$ derived from interferometric observations by Dyck et al. ${ }^{47}$ and $T_{\text {eff }}=3978$ $\pm 46 \mathrm{~K}$ presented in ref. ${ }^{46}$. For five common stars, our $\log g$ was found to be slightly lower by $\sim 0.40$ dex compared to ref. ${ }^{46}$ (Supplementary Fig. $5 c$, d), however still in a good agreement within $3 \sigma$.

We conclude that the presented method is sensitive to effective temperature and surface gravity and can be used for determining $T_{\text {eff }}$ and $\log g$ for giants and supergiant stars of $\mathrm{K}$ - and $\mathrm{M}$-spectral types based on their high-resolution spectral observations in the near-IR spectral range.

\section{Data availability}

All data generated and/or analyzed during the current study are available from the corresponding author upon request. Source data are provided with this paper.

\section{Code availability}

Computer MCMC code is available from GitHub under https://github.com/salex-asto/ Betelgeuse-NC. We made use of MARCS ${ }^{32}$, SYNTHV_NLTE ${ }^{34}$, DETAIL ${ }^{35}$, EMCEE package ${ }^{41}$, BINMAG v.6 code [http://www.astro.uu.se/ oleg/binmag.html $]^{37}$

Received: 9 July 2020; Accepted: 14 July 2021; Published online: 05 August 2021

\section{References}

1. Montargès, M., Cannon, E., Kervella, P. \& Ferreira, B. ESO Press Release ESO2003. https://www.eso.org/public/news/eso2003/ (2020).

2. Levesque, E. M. \& Massey, P. Betelgeuse just is not that cool: effective temperature alone cannot explain the recent dimming of Betelgeuse. Astrophys. J. Lett. 891, L37 (2020).

3. Gupta, A. \& Sahijpal, S. Thermodynamics of dust condensation around the dimming Betelgeuse. Month. Notices RAS 496, L122 (2020).

4. Dupree, A. K. et al. Spatially resolved ultraviolet spectroscopy of the great dimming of Betelgeuse. Astrophys. J. 899, 68 (2020).

5. Harper, G. M. et al. SOFIA-EXES observations of Betelgeuse during the great dimming of 2019/2020. Astrophys. J. Lett. 893, L23 (2020).

6. Harper, G. M., Guinan, E. F., Wasatonic, R. \& Ryde, N. The photospheric temperatures of Betelgeuse during the great dimming of 2019/2020: no new dust required. Astrophys. J. 905, 34 (2020).

7. Dharmawardena, T. E. et al. Betelgeuse fainter in the submillimeter too: an analysis of JCMT and APEX monitoring during the recent optical minimum. Astrophys. J. Lett. 897, L9 (2020).

8. George, S. V., Kachhara, S., Misra, R. \& Ambika, G. Early warning signals indicate a critical transition in Betelgeuse. Astron. Astrophys. 640, L21 (2020)

9. Levesque, E. M. et al. The effective temperatures and physical properties of magellanic cloud red supergiants: the effects of metallicity. Astrophys. J. 645, 1102 (2006).

10. Gustafsson, B., Bell, R. A., Eriksson, K. \& Nordlund, A. Reprint of 1975A\&A...42 ...407G. A grid of model atmospheres for metal-deficient giant stars. I. Astron. Astrophys. 500, 67 (1975).

11. Gustafsson, B. et al. in Stellar Atmosphere Modeling, Astronomical Society of the Pacific Conference Series (eds Hubeny, I., Mihalas, D. \& Werner, K.) Vol. 288, 331 (2003).

12. Plez, B. Spherical opacity sampling model atmospheres for M-giants and supergiants. II. A grid. Astron. Astrophys. Suppl. 94, 527 (1992).

13. Plez, B. in GAIA Spectroscopy: Science and Technology, Astronomical Society of the Pacific Conference Series (ed. Munari, U.) Vol. 298, 189 (2003).

14. Arroyo-Torres, B., Wittkowski, M., Marcaide, J. M. \& Hauschildt, P. H. The atmospheric structure and fundamental parameters of the red supergiants $\mathrm{AH}$ Scorpii, UY Scuti, and KW Sagittarii. Astron. Astrophys. 554, A76 (2013).

15. Levesque, E. M. et al. The effective temperature scale of galactic red supergiants: cool, but not as cool as we thought. Astrophys. J. 628, 973 (2005).

16. Levesque, E. M. Astrophysics of Red Supergiants (IOP Publishing, Bristol, UK, 2017).

17. Wilson, R. W., Baldwin, J. E., Buscher, D. F. \& Warner, P. J. High-resolution imaging of Betelgeuse and Mira. Month. Notices RAS 257, 369 (1992).

18. Wilson, R. W., Dhillon, V. S. \& Haniff, C. A. The changing face of Betelgeuse. Month. Notices RAS 291, 819 (1997).

19. Young, J. S. et al. New views of Betelgeuse: multi-wavelength surface imaging and implications for models of hotspot generation. Month. Notices RAS 315, 635 (2000).

20. Strassmeier, K. G. Starspots. Astron. Astrophys. 17, 251 (2009).

21. Montargès, M. et al. The convective photosphere of the red supergiant CE Tauri. I. VLTI/PIONIER H-band interferometric imaging. Astron. Astrophys. 614, A12 (2018).

22. Schwarzschild, M. On the scale of photospheric convection in red giants and supergiants. Astrophys. J. 195, 137 (1975).

23. Doherty, L. R. On the polarization of alpha orionis. Astrophys. J. 307, 261 (1986).

24. Buscher, D. F., Haniff, C. A., Baldwin, J. E. \& Warner, P. J. Detection of a bright feature on the surface of Betelgeuse. Month. Notices RAS 245, p. 7P (1990).

25. Tuthill, P. G., Haniff, C. A. \& Baldwin, J. E. Hotspots on late-type supergiants. Month. Notices RAS 285, 529 (1997).

26. Haubois, X. et al. Imaging the spotty surface of Betelgeuse in the $\mathrm{H}$ band. Astron. Astrophys. 508, 923 (2009). 
27. Kervella, P. et al. The close circumstellar environment of Betelgeuse. V. Rotation velocity and molecular envelope properties from ALMA. Astron. Astrophys. 609, p. A67 (2018).

28. Uitenbroek, H., Dupree, A. K. \& Gilliland, R. L. Spatially resolved Hubble space telescope spectra of the chromosphere of alpha Orionis. Astron. J. 116, 2501 (1998).

29. Gao, D.-Y. et al. WES-Weihai Echelle spectrograph. Public. ASP 128, 125002 (2016).

30. Hu, S.-M., Han, S.-H., Guo, D.-F. \& Du, J.-J. The photometric system of the one-meter telescope at Weihai Observatory of Shandong University. Res. Astron. Astrophys. 14, 719 (2014).

31. Donati, J.-F., Catala, C., Landstreet, J. D. \& Petit, P. in Solar Polarization 4, Astronomical Society of the Pacific Conference Series (eds Casini, R. \& Lites, B. W.) Vol. 358, 362 (2006).

32. Gustafsson, B. et al. A grid of MARCS model atmospheres for late-type stars. I. Methods and general properties. Astron. Astrophys. 486, 951 (2008).

33. Ryabchikova, T. et al. A major upgrade of the VALD database. Phys. Scr. 90, 054005 (2015)

34. Ryabchikova, T. et al. Accuracy of atmospheric parameters of FGK dwarfs determined by spectrum fitting. Month. Notices RAS 456, 1221 (2016).

35. Butler, K. \& Giddings, J. Newsletter on the Analysis of Astronomical Spectra Vol. 9 (University of London, 1985).

36. Rybicki, G. B. \& Hummer, D. G. An accelerated lambda iteration method for multilevel radiative transfer. I Non overlapping lines with background continuum. Astron. Astrophys. 245, 171 (1991).

37. Kochukhov, O. BinMag: widget for comparing stellar observed with theoretical spectra. Astrophysics Source Code Library, http://www.astro.uu.se/ $\sim$ oleg/binmag.html (2018).

38. Kurucz, R. L., Furenlid, I., Brault, J. \& Testerman, L. Solar Flux Atlas from 296 to $1300 \mathrm{~nm}$ (National Solar Observatory, National Solar Observatory Atlas, Sunspot, New Mexico, 1984).

39. Lambert, D. L., Brown, J. A., Hinkle, K. H. \& Johnson, H. R. Carbon, nitrogen and oxygen abundances in Betelgeuse. Astrophys. J. 284, 223 (1984).

40. Naulin, C., Hedgecock, I. M. \& Costes, M. The dissociation energy of TiO determined from a crossed-beam study of the $\mathrm{Ti}+\mathrm{NO}-\mathrm{TiO}+\mathrm{N}$ reaction. Chem. Phys. Lett. 266, 335 (1997).

41. Foreman-Mackey, D., Hogg, D. W., Lang, D. \& Goodman, J. emcee: the MCMC hammer. Publications of the ASP 125, 306 (2013).

42. Bauschlicher, J., Charles, W., Langhoff, S. R. \& Taylor, P. R. Theoretical study of the dissociation energy and the red and violet band systems of $\mathrm{CN}$. Astrophys. J. 332, 531 (1988).

43. Alexeeva, S. A., Pakhomov, Y. V. \& Mashonkina, L. I. Non-LTE sodium abundance in galactic thick- and thin-disk red giants. Astron. Lett. 40, 406 (2014).

44. Alexeeva, S., Ryabchikova, T., Mashonkina, L. \& Hu, S. NLTE line formation for $\mathrm{Mg} \mathrm{I}$ and $\mathrm{Mg}$ II in the atmospheres of B-A-F-G-K stars. Astrophys. J. 866, 153 (2018).

45. McDonald, I., Zijlstra, A. A. \& Boyer, M. L. Fundamental parameters and infrared excesses of Hipparcos stars. Month. Notices RAS 427, p. 343 (2012).

46. Prugniel, P., Vauglin, I. \& Koleva, M. The atmospheric parameters and spectral interpolator for the MILES stars. Astron. Astrophys. 531, p. A165 (2011).

47. Dyck, H. M., van Belle, G. T. \& Thompson, R. R. Radii and effective temperatures for K and M giants and supergiants. II. Astron. J. 116, 981 (1998).

\section{Acknowledgements}

This study is supported by the National Natural Science Foundation of China under grants Nos. 11988101, 11890694, 12050410265, and National Key R\&D Program of China No. 2019YFA0405502. This research is based on spectroscopic observations carried out at the Weihai Observatory of Shandong University in the People's Republic of China. S.A. acknowledges support from the LAMOST FELLOWSHIP that is budgeted and administrated by the Chinese Academy of Sciences. This research used the facilities of the Canadian Astronomy Data Centre operated by the National Research Council of Canada with the support of the Canadian Space Agency. We acknowledge with thanks the variable star observations from the AAVSO International Database contributed by observers worldwide and used in this research. We made use of NIST, SIMBAD, and VALD databases. Based on observations collected at the European Southern Observatory under ESO program 266.D-5655(A).

\section{Author contributions}

G.Z. and S.A. proposed and designed this study. K.L. and S.H. provided observational time and organized the high-resolution spectroscopic observations. D.-Y.G. carried out the observations and made data reduction. A.L. participated in the discussion on the possible obscuring effects of dust. S.A. applied the theoretical methods and led the data analysis. J.D. developed MCMC Python code. All the authors discussed the results and contributed to the writing of the manuscript.

\section{Competing interests}

The authors declare no competing interests.

\section{Additional information}

Supplementary information The online version contains supplementary material available at https://doi.org/10.1038/s41467-021-25018-3.

Correspondence and requests for materials should be addressed to G.Z.

Peer review information Nature Communications thanks, Anuj Gupta, and the other, anonymous, reviewer(s) for their contribution to the peer review of this work.

Reprints and permission information is available at http://www.nature.com/reprints

Publisher's note Springer Nature remains neutral with regard to jurisdictional claims in published maps and institutional affiliations.

\begin{abstract}
(c) (i) Open Access This article is licensed under a Creative Commons BY Attribution 4.0 International License, which permits use, sharing, adaptation, distribution and reproduction in any medium or format, as long as you give appropriate credit to the original author(s) and the source, provide a link to the Creative Commons license, and indicate if changes were made. The images or other third party material in this article are included in the article's Creative Commons license, unles indicated otherwise in a credit line to the material. If material is not included in the article's Creative Commons license and your intended use is not permitted by statutory regulation or exceeds the permitted use, you will need to obtain permission directly from the copyright holder. To view a copy of this license, visit http://creativecommons.org/ licenses/by/4.0/.
\end{abstract}

(c) The Author(s) 2021, corrected publication 2021 